\title{
Surface and Core Detonations in Rotating White Dwarfs
}

\author{
D. García-Senz ${ }^{1,2}$ (10), R. M. Cabezón ${ }^{3}$ (1), and I. Domínguez ${ }^{4}$ (1) \\ ${ }^{1}$ Departament de Física, UPC, (EEBE) Avenida Eduard Maristany 16, E-08019 Barcelona, Spain; domingo.garcia@upc.edu \\ ${ }^{2}$ Institut d'Estudis Espacials de Catalunya, Gran Capità 2-4, E-08034 Barcelona, Spain \\ 3 sciCORE, Universität Basel. Klingelbergstrasse, 61, 4056 Basel, Switzerland; ruben.cabezon@unibas.ch \\ ${ }^{4}$ Departamento de Física Teórica y del Cosmos, Universidad de Granada, E-18071 Granada, Spain \\ Received 2018 January 29; revised 2018 May 25; accepted 2018 June 6; published 2018 July 19
}

\begin{abstract}
The feasibility of the double detonation mechanism—surface helium detonation followed by complete carbon detonation of the core-in a rotating white dwarf with mass $\simeq 1 M_{\odot}$ is studied using three-dimensional hydrodynamic simulations. A rapid rigid rotation of the white dwarf was assumed, so that its initial spherical geometry is considerably distorted. Unlike spherically symmetric models, we found that when helium ignition is located far from the spinning axis, the detonation fronts converge asynchronically at the antipodes of the ignition point. Nevertheless, the detonation of the carbon core still remains as the most probable outcome. The detonation of the core gives rise to a strong explosion, matching many of the basic observational constraints of Type Ia supernovae ( $\mathrm{SNe}$ Ia). We conclude that the double detonation mechanism also works when the white dwarf is rapidly rotating. These results provide further evidence for the viability of sub-Chandrasekhar-mass models as well as some double degenerate models (those having some helium fuel at the merging moment), making them appealing channels for the production of SN Ia events.
\end{abstract}

Key words: hydrodynamics - methods: numerical - nuclear reactions, nucleosynthesis, abundances - stars: rotation supernovae: general - white dwarfs

\section{Introduction}

A challenging task in astrophysics is to unveil the progenitors and explosion mechanisms of Type Ia supernovae (SNe Ia). Nowadays, observational and theoretical arguments point to two major production channels for these explosions, the single degenerate (SD; Whelan \& Iben 1973) and double degenerate (DD; Iben \& Tutukov 1984) scenarios (for reviews, see, e.g., Hillebrandt et al. 2013; Maoz et al. 2014). The precise fraction of SNe Ia coming from each channel is still a matter of vigorous debate.

A particular class of SD models that has recently received attention is the double detonation (DDet) scenario. In the DDet model, a carbon-oxygen (CO) white dwarf (WD) with masses $\simeq 0.8-1.1 M_{\odot}$ incorporates helium through accretion from a companion star. Under the appropriate conditions (Woosley \& Weaver 1994), the helium detonates above the edge of the CO core, which in turn induces a second detonation of carbon, thus producing an SN Ia.

There was a time when these sub-Chandrasekhar-mass explosion models (hereafter, subCh-mass models) had some success. They were able to reproduce many supernova observables, especially the explosion energy and gross nucleosynthetic production in subluminous events (Woosley et al. 1986; Woosley \& Weaver 1994; Livne \& Arnett 1995; García-Senz et al. 1999). At the same time, the DDet explosion mechanism (Livne \& Glasner 1991) was understood better than the subsonic deflagration that powers, at least initially, the explosion in the Chandrasekhar-mass models (Nomoto et al. 1984; Khokhlov 1991; Hillebrandt \& Niemeyer 2000). Nevertheless, the subCh-mass explosion models suffer from several drawbacks. The most acute of these is that the synthetic spectra do not match observations because they predict too much high-velocity ${ }^{56} \mathrm{Ni}$ in the external layers, which also produce blue colors at maximum light due to radioactive heating, in contrast with observations (Hoeflich et al. 1996; Nugent et al. 1997).

The situation changed when it was realized that the DDet mechanism could be at work even in helium layers as thin as $\simeq 10^{-2} M_{\odot}$ (Bildsten et al. 2007), so the nickel problem vanishes. Recent investigations of detonations in helium shells and their nucleosynthetic outcomes have been conducted by Moore et al. (2013) and Shen \& Moore (2014). It was also realized that the observed $\mathrm{SNe}$ Ia rates and delayed time distributions could not be reproduced assuming only SD and DD Chandrasekhar-mass explosions, although including SD and DD subCh-mass explosions may solve the problem (Badenes \& Maoz 2012; Ruiter et al. 2011; Maoz et al. 2014). Moreover, it has been recently claimed (Blondin et al. 2017; Goldstein \& Kasen 2018) that the faint end of the Phillips relation (Phillips 1993; Phillips et al. 1999) could only be reproduced with subCh-mass explosions (but see also Hoeflich et al. 2017).

Recent multidimensional simulations of the DDet scenario have been carried out by Sim et al. (2007, 2010, 2012) and Fink et al. $(2007,2010)$ in two dimensions (ignition in a point makes the problem axisymmetric) as well as in three dimensions (Moll \& Woosley 2013), in this last case to discern the outcome of multipoint ignitions. All of them concluded that the DDet mechanism is robust, being able to successfully cope with a variety of helium-shell masses and symmetric and nonsymmetric initial conditions.

Despite the fact that accretion or merging scenarios imply, up to some degree, rotation of the exploding WD, the number of SD calculations that incorporate the effects of rotation in the explosion is really low. Rapidly spinning WDs with masses $1.46 M_{\odot} \leqslant M_{\mathrm{WD}} \leqslant 2.02 M_{\odot}$ were considered by Pfannes et al. (2010a, 2010b), who tried to explain the differences in the peak luminosity as a function of the rotation strength. They concluded, however, that the match of the deflagration models 
with observations becomes worse for rotating WDs. Conversely, if the star explodes following a detonation, superChandrasekhar-mass models in fast rotation may explain some basic features of superluminous Type Ia events. The impact of a moderate amount of rotation on the gravitationally confined detonation (GCD) model (Plewa et al. 2004) has been explored by García-Senz et al. (2016), who concluded that rotation is a necessary ingredient to discern whether or the $\mathrm{CO}$ core detonates.

In this work, we investigate, for the first time, the feasibility of the DDet mechanism when a WD with mass $\simeq 1 M_{\odot}$ is rotating rapidly. This is especially relevant in this case because the secondary detonation of the $\mathrm{CO}$ core requires the focusing of the shock waves produced during the He-shell detonation onto a small region at the symmetry axis. We investigate to what extent such wave convergence might be hampered in rotating models, especially when the helium ignition takes place in a point-like region far from the spinning axis. Additionally, our models predict several properties that could be compared with observations, like kinetic energies, nuclear yields, and asymmetries produced by the explosion mechanism.

In Section 2, we describe the main features of the spinning WDs considered in this work. In Section 3, we comment on the main features of the hydrodynamics code (SPHYNX) used in this work, the initial setting, and the method for building stable rotating WDs in rigid rotation (this is described in more detail in the Appendix). We give a detailed description of the hydrodynamic evolution and nucleosynthesis during the detonation of the helium shell in Section 4. The detonation of the core and its consequences are described in Section 5. Finally, Section 6 summarizes the main conclusions of our work.

\section{Rotation of Accreting White Dwarfs}

The conservation of angular momentum makes compact objects prone to having large spinning velocities. In particular, for compact binary systems, the rotational velocity of the accreting WD benefits from the transfer of angular momentum from the accretion disk, which is even able to approach the centrifugal threshold (Yoon \& Langer 2004b). In the case of subCh-mass models of SNe Ia, an upper limit of the rotation velocity can be inferred by assuming that the angular momentum of the accreted shell is efficiently transferred to the underlying WD. Thus, considering no angular momentum losses, a quantitative relationship between the amount of accreted matter and the normalized angular velocity, $\Omega=\omega_{\text {acc }} / \omega_{\text {kepl }}$, of the WD can be established (Langer et al. 2000),

$$
\Omega=\frac{3}{4 r_{g}^{2}}\left[1-\left(\frac{M_{\mathrm{WD}, \mathrm{i}}}{M_{\mathrm{WD}}}\right)^{\frac{4}{3}}\right],
$$

where $M_{\mathrm{WD}, \mathrm{i}}$ is the initial mass of the WD prior to accretion, $M_{\mathrm{WD}}$ is the mass of the WD, $\omega_{\mathrm{acc}}$ is the angular velocity gained from the accretion disk, $\omega_{\mathrm{Kepl}}$ is the Keplerian angular velocity, and $r_{g}$ is the gyration radius (Ritter 1985),

$$
r_{g}=0.452+0.0853 \log \left(1-\frac{M_{\mathrm{WD}}}{M_{\mathrm{CH}}}\right) ; \quad M_{\mathrm{WD}} \leqslant 0.95 M_{\mathrm{CH}},
$$

where $M_{\mathrm{CH}}$ is the Chandrasekhar-mass limit. The Keplerian velocity is

$$
\omega_{\mathrm{Kepl}}=\sqrt{\frac{G M_{\mathrm{WD}}}{R^{3} \mathrm{WD}}} .
$$

According to the published literature on subCh-mass models, the thickness of the helium shell, $\Delta M_{\mathrm{He}}$, at the moment of explosion is within the range $0.01 M_{\odot} \leqslant \Delta M_{\mathrm{He}} \leqslant 0.15 M_{\odot}$ (Fink et al. 2010; Sim et al. 2012; Moll \& Woosley 2013). Considering $M_{\mathrm{WD}}=1 \quad M_{\odot}$ and $M_{\mathrm{WD}, \mathrm{i}}=0.85 \quad M_{\odot} \quad$ in Equation (1), it results in $\Omega \simeq 0.9$. Such a large value would bring the WD close to its centrifugal limit, and as a consequence, the initially spherical geometry will evolve into an oblate spheroid, which may have an impact on the outcome of the explosion.

A heuristic calculation may help select the adequate candidates for the hydrodynamic simulations of surface detonations in rotating WDs. First, we set the minimum density, $\rho_{\mathrm{He}}$, able to support a steady helium detonation. According to previous studies, $\rho_{\mathrm{He}} \geqslant 10^{6} \mathrm{~g} \mathrm{~cm}^{-3}$ (Woosley \& Weaver 1994; Woosley \& Kasen 2011; Holcomb et al. 2013; Moll \& Woosley 2013). Nonetheless, the precise value of such a minimum ignition density is not a well-constrained magnitude. It may depend, among other things, on the extent of the ${ }^{12} \mathrm{C} /{ }^{16} \mathrm{O} /{ }^{14} \mathrm{~N}$ pollution in the He layer, on the precise temperature profile around the hot spot, and on the size of the reaction network used in the calculation (Shen \& Moore 2014). We choose $\rho_{\mathrm{He}}=1.6 \times 10^{6} \mathrm{~g} \mathrm{~cm}^{-3}$ as the nominal density at the core-envelope interface at the moment of explosion, following Moll \& Woosley (2013). We then integrate the structure equations of a WD for a grid of central densities in the range $10^{7} \leqslant \rho_{c} \leqslant 4 \times 10^{8} \mathrm{~g} \mathrm{~cm}^{-3}$ at a constant temperature of $10^{6} \mathrm{~K}$, and we switch the chemical composition from $X_{\mathrm{C}}=X_{\mathrm{O}}=0.5$ to $X_{\mathrm{He}}=1$ when $\rho \leqslant \rho_{\mathrm{He}}$. Such a switch marks the edge between the $\mathrm{CO}$ core and the He envelope. In this rough approach, $M_{\mathrm{WD}}, \Delta M_{\mathrm{He}}, \Omega$, and $\omega_{\mathrm{Kepl}}$, depend exclusively on the adopted central density at the moment of explosion.

The ensuing grid of models is depicted in Figure 1, where the upper panel gives the profile of $\Delta M_{\mathrm{He}}$ and $M_{\mathrm{WD}}$ as a function of the central density, while the lower panel presents information concerning the angular velocity. As can be seen, the profile of $\Delta M_{\mathrm{He}}$ is no longer linear. Tiny He envelopes $\left(\simeq 0.02 M_{\odot}\right)$ would require rather massive WD cores $\left(\simeq 1.2 M_{\odot}\right)$, or equivalently, large WD masses prior to accretion. On the contrary, thick He layers $\left(\simeq 0.10 M_{\odot}\right)$ would require a less massive WD $\left(\simeq 0.8 M_{\odot}\right)$ prior to accretion. Such profile follows approximately the $\left(\rho_{c}, \Delta M_{\mathrm{He}}\right)$ relationship inferred from the data by Fink et al. (2010) and Moll \& Woosley (2013; triangles and crosses in Figure 1, respectively).

The lower panel in Figure 1 depicts the angular velocity, $\omega_{\text {acc }}$, of the WD after accreting $\Delta M_{\mathrm{He}}$ and the Keplerian velocity $\omega_{\mathrm{Kepl}}$, as well as their ratio $\Omega=\omega_{\text {acc }} / \omega_{\mathrm{Kepl}}$. Any physically sound value of $\omega$ has to fulfill $\omega \leqslant \omega_{\text {acc }} \leqslant \omega_{\text {Kepl }}$, where the equality $\omega=\omega_{\text {acc }}$ stands for conservative angular momentum transfer from the disk to the WD. The symbols $\oplus$ in the figure indicate the location of models A, B, C, and $\mathrm{D}$ described in Table 1. Models $\mathrm{A}$ and $\mathrm{C}$ are non-rotating models, while $\mathrm{B}$ and $\mathrm{D}$ are the corresponding rotating versions. We see that the angular velocity of model $B$ is in the region of interest of the diagram, albeit close to $\omega_{\text {acc }}$. It is also worth 


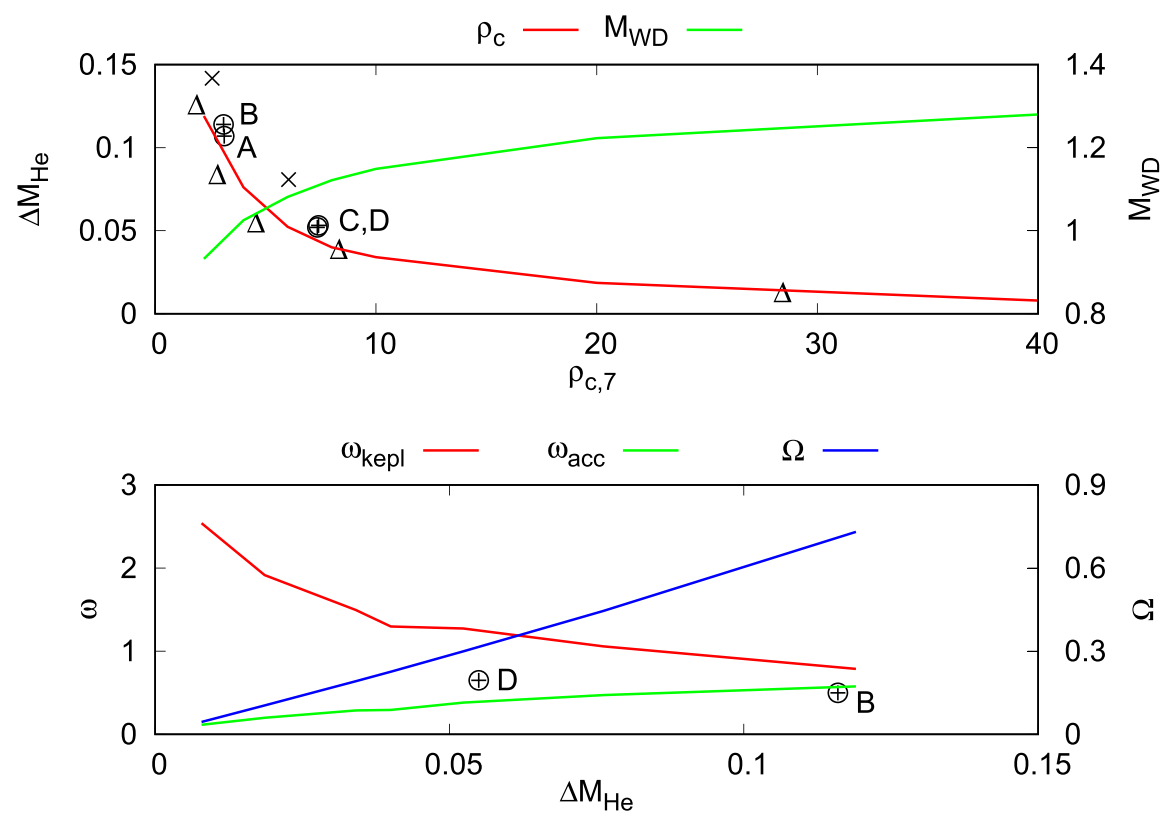

Figure 1. Upper panel: mass of the helium shell $\left(\Delta M_{\mathrm{He}}\right.$, in $\left.M_{\odot}\right)$ on top of a $\mathrm{CO}$ core as a function of the central density for spherically symmetric models. Lower panel: rotational angular velocity of the $\mathrm{WD}\left(\mathrm{s}^{-1}\right)$ as a function of the mass of the helium-shell envelope. Symbols $\Delta, \times$, and $\oplus$ refer to the explosion models reported in Fink et al. (2010), Moll \& Woosley (2013), and Table 1 in this work (models A, B, C, and D), respectively.

noting that although the rotation of model $D$ is neatly subKeplerian, its angular velocity is slightly above the $\omega_{\text {acc }}$ line.

WDs are very compact and chemically homogeneous objects, so the transport of angular momentum is expected to be very efficient (Maeder \& Meynet 2000; Piro 2008; Saio \& Nomoto 2004) and the accreting WD may be treated as a rigid rotator. The presence of magnetic fields favors rigid rotation (Neunteufel et al. 2017), although for non-magnetic subChandrasekhar masses, the final rotational state is not as well constrained (Ghosh \& Wheeler 2017) and differential rotating WDs may end as helium novae (Yoon \& Langer 2004a). We decide to adopt a practical approach and assume rigid rotation in all our models. Models $B$ and $D$ therefore represent extreme cases in the sense that if the DDet mechanism works for them, it will also work for any rotating model located below the $\omega_{\text {acc }}$ line in Figure 1. Additionally, the minimum observed period of a WD in a cataclysmic variable is $P=27.8 \mathrm{~s}$ for WZ Sag (Patterson 1980). That period is larger than the value $P \simeq 5 \mathrm{~s}$ obtained with Equations (1)-(3), with $M_{\mathrm{WD}}{ }^{i}=$ $0.85 M_{\odot}, M_{\mathrm{WD}}=1 M_{\odot}$ and $R_{\mathrm{WD}}=5000 \mathrm{~km}$, suggesting a non-conservative evolution during the accretion. Some fraction of the incoming angular momentum is probably lost during the recurrent, nova-like, phenomena associated with the surface flashes which transform the accreted hydrogen into helium. Also, the polarization spectra of common (branch-normal) SN Ia explosions do not favor large departures from spherical geometry (Wang \& Wheeler 2008). All of these suggest that the rigid body angular velocity obtained using Equations (1) and (3) has to be taken as an upper limit. In this regard, we note that high, near-Keplerian rotational velocities may be achieved during the merging process of two WDs in the DD scenario (Lorén-Aguilar et al. 2009; Dan et al. 2015).

Additionally, the rotational velocities considered in models $\mathrm{B}$ and $\mathrm{D}$ in Table 1 are high enough to leave some imprint on the geometry and distribution of mass within the WDs. In that case, if the explosion mechanism and the main observables of the explosion do not appreciably differ from the spherically symmetric case, we can safely infer that rotation does not represent a problem for the viability of SNe Ia subCh-mass models.

\section{Hydrodynamic Method and Initial Setting}

Surface He detonations on top of massive rotating $\mathrm{CO}$ cores $\left(\geqslant 0.8 M_{\odot}\right)$ are an intrinsic $3 \mathrm{D}$ phenomena. During the explosion, the former helium shell is ejected with velocities $\geqslant 2 \times 10^{4} \mathrm{~km} \mathrm{~s}^{-1}$, so that the characteristic size of the object changes from the initial $R \simeq 5 \times 10^{3} \mathrm{~km}$ to $\simeq 10^{5} \mathrm{~km}$ over a few seconds. Such a large change in size, along with the multidimensional nature of the explosion, makes Lagrangian methods, such as smoothed particle hydrodynamics (SPH), ideally suited for simulating these systems. Moreover, the addition of rotation renders this problem difficult to study using Eulerian hydrodynamics. To carry out the simulations, we made use of the Integral-SPH (ISPH) hydrocode SPHYNX (Cabezón et al. 2017), conveniently adapted to handle explosive scenarios involving degenerate matter (García-Senz et al. 2016). SPHYNX is a state-of-the-art hydrocode with an improved algorithm for estimating gradients, which relies on an integral approach (García-Senz et al. 2012) to the derivatives. It also makes use of the sinc family of kernels (Cabezón et al. 2008), which is resistant to particle clustering, therefore allowing the number of interpolating particles in the SPH summations to increase in order to reduce the numerical noise.

The physical processes included are very similar to those recently used by García-Senz et al. (2016) to study the GCD explosion mechanism. An efficient nuclear network evaluates the energy input and rough composition change due to nuclear reactions via an $\alpha$-chain with 14 species from ${ }^{4} \mathrm{He}$ to ${ }^{60} \mathrm{Zn}$. The alpha network is completed with the reactions ${ }^{12} \mathrm{C}+{ }^{12} \mathrm{C},{ }^{12} \mathrm{C}$ $+{ }^{16} \mathrm{O}$, and ${ }^{16} \mathrm{O}+{ }^{16} \mathrm{O}$. Effective hidden rates linked to proton reactions are not included in the network. The evolution of the species is calculated implicitly and coupled with the temperature to ensure a smooth transition to the nuclear-statistical equilibrium 
Table 1

Main Features of the Initial Models

\begin{tabular}{lccccccccccc}
\hline \hline Model & $\begin{array}{c}N \\
10^{6} \mathrm{part}\end{array}$ & $\begin{array}{c}\text { Ignition Altitude } \\
\mathrm{km}\end{array}$ & $\begin{array}{c}\omega_{x} \\
\mathrm{~s}^{-1}\end{array}$ & Ignition axis & $\begin{array}{c}\rho_{c} \\
10^{7} \mathrm{~g} \mathrm{~cm}^{-3}\end{array}$ & $\begin{array}{c}\rho_{\mathrm{He}} \\
10^{7} \mathrm{~g} \mathrm{~cm}^{-3}\end{array}$ & $\begin{array}{c}h_{c} \\
\mathrm{~km}\end{array}$ & $\begin{array}{c}h_{\mathrm{He}} \\
\mathrm{km}\end{array}$ & $\begin{array}{c}M_{\mathrm{WD}} \\
M_{\odot}\end{array}$ & $\begin{array}{c}\Delta M_{\mathrm{He}} \\
M_{\odot}\end{array}$ & $\begin{array}{c}\text { Oblateness } \\
f\end{array}$ \\
\hline $\mathrm{A}_{1}$ & 2.0 & 4200 & 0.00 & $X$ & 2.60 & 0.15 & 48 & 129 & 0.9590 & 0.1068 \\
$\mathrm{~B}_{1}$ & 2.0 & 4300 & 0.50 & $X$ & 2.57 & 0.11 & 49 & 142 & 1.0815 & 0.1140 \\
$\mathrm{~B}_{2}$ & 2.0 & 4550 & 0.50 & $X Y$ & 2.57 & 0.11 & 49 & 142 & 1.0815 & 0.1140 & 0.35 \\
$\mathrm{~B}_{3}$ & 2.0 & 5000 & 0.50 & $Y$ & 2.57 & 0.11 & 49 & 142 & 1.0815 & 0.1140 & 0.35 \\
$\mathrm{~B}_{4}$ & 2.0 & 3900 & 0.50 & $X$ & 2.57 & 0.15 & 49 & 129 & 1.0815 & 0.1533 & 0.35 \\
$\mathrm{~B}_{5}$ & 2.0 & 4200 & 0.50 & $X Y$ & 2.57 & 0.15 & 49 & 129 & 1.0815 & 0.1533 \\
$\mathrm{~B}_{6}$ & 2.0 & 4600 & 0.50 & $Y$ & 2.57 & 0.15 & 49 & 129 & 1.0815 & 0.1533 & 0.35 \\
$\mathrm{C}_{1}$ & 4.0 & 3880 & 0.00 & $X$ & 6.82 & 0.15 & 29 & 102 & 1.1052 & 0.0520 & 0.00 \\
$\mathrm{D}_{1}$ & 4.0 & 3700 & 0.65 & $X$ & 6.87 & 0.12 & 30 & 113 & 1.1872 & 0.0532 \\
$\mathrm{D}_{2}$ & 4.0 & 4230 & 0.65 & $Y$ & 6.87 & 0.12 & 30 & 113 & 1.1872 & 0.0532 \\
\hline
\end{tabular}

Note. Columns show model name, number of particles, initial bubble ignition altitude with respect to the center of the WD, angular velocity, location of the initial bubble ( $X Y$ refers to an ignition at $45^{\circ}$ in the $X-Y$ plane), central density of the WD, density at the He-core interface, shortest smoothing length (i.e., highest spatial resolution) at the core and at the He layer, total mass of the WD (CO core + He envelope), mass of the He envelope, and oblateness factor as $f=(a-b) / a$, where $a \simeq 8000 \mathrm{~km}$ and $b \simeq 5200 \mathrm{~km}$ are the equatorial and polar radius in the B models. The radius of the igniting ball at the edge of the core is $R_{b}=250 \mathrm{~km}$ in all models.

(NSE) regime (Cabezón et al. 2004). Although it is computationally very expensive to handle the nuclear reactions with a nuclear network for $T>4 \times 10^{9} \mathrm{~K}$, we have taken that approach because the densities are not high enough to assume complete NSE. Also, our scheme provides a good approach to the nucleosynthetic yields during the freeze-out of the reactions. Electron captures on protons and nuclei have been neglected because their impact on the dynamics of the explosion is secondary. Note that central densities are more than two orders of magnitude lower than explosion ignition densities in Chandrasekhar-mass WDs. Our EOS has the contributions from electrons (Blinnikov et al. 1996), ions (including Coulomb and polarization corrections), and radiation.

All calculations reported in this paper assume that the thermonuclear ignition of the WD starts in a single spherical region located in the helium-rich region, close to the core -envelope edge. ${ }^{5}$ Ideally, the size of such an initial detonator is dictated by the environmental physical conditions set during the pre-ignition state, especially by peak density and temperature values and profiles. However, current three-dimensional calculations do not have sufficient resolution to allow a selfconsistent initiation of the explosion, and therefore, helium detonation must be artificially triggered.

\subsection{Implementation of Rotation}

An accurate method to build rotating WDs in hydrostatic equilibrium within the SPH framework does not exist. We have developed and checked a relaxation procedure that is able to produce self-gravitating rotating WDs in equilibrium. This topic is, by itself, of sufficient interest for the SPH community as to deserve careful description and analysis, which are deferred to an upcoming publication. Nevertheless, the foundations of the method are described in the Appendix, where we provide the reader with some details on how we built the stable, rigidly rotating WDs considered in this work.

\footnotetext{
Moll \& Woosley (2013) also explored the impact of starting the $\mathrm{He}$ detonation at some altitude above the interface, when the density is $\rho_{\mathrm{He}} \simeq 1.6 \times 10^{6} \mathrm{~g} \mathrm{~cm}^{-3}$.
}

\section{Hydrodynamic Simulations: The He-shell Detonation}

In this section, we address the helium-shell detonation independently of the core detonation. The feasibility of having the $\mathrm{CO}$ core detonate right after the explosion of the helium envelope is also analyzed. Although the simulations suggest that the detonation in the $\mathrm{CO}$ core is the most probable outcome, we have also obtained the basic observational properties of $\mathrm{He}$ detonation alone. When the heliumshell detonation is not followed by the detonation of the core, the resulting event is a dim supernova, whose main features are presented and discussed in the following subsections. The description of the hydrodynamic and nuclear evolution of the complete explosion of the WD is left to Section 5.

\subsection{Evolution of the Reference Model}

Our control model is $A_{1}$, in Table 1. This is a non-rotating spherically symmetric model of a WD with $M_{\mathrm{WD}}=$ $0.9590 M_{\odot}$ and $\Delta M_{\mathrm{He}}=0.107 M_{\odot}$. On the other hand, our reference models $\left(\mathrm{B}_{1}, \mathrm{~B}_{2}\right.$, and $\left.\mathrm{B}_{3}\right)$ for rotating WDs have a total mass $M_{\mathrm{WD}}=1.0815 M_{\odot}$. The helium shell amounts to $\Delta M_{\mathrm{He}}=0.114 M_{\odot}$, similar to that of the spherically symmetric model $\mathrm{A}_{1}$. In the $\mathrm{B}$ models, the WD is rotating as a rigid body around the $X$-axis, with a value $\omega_{x}=0.5 \mathrm{~s}^{-1}$. As quoted before, we have decided to explore an upper limit in terms of rotational velocity. We note that a non-magnetic massive WD with this high angular velocity has been observed (Mereghetti 2015; Popov et al. 2018) in a binary system, although the origin of such rotation is still unclear. The unique difference among $\mathrm{B}$ models is the location where the He detonation starts: aligned $\left(B_{1}\right)$, or at $45^{\circ}\left(B_{2}\right)$ or $90^{\circ}\left(B_{3}\right)$ with respect to the rotation axis. The outcomes of these calculations are compared to the control model $\mathrm{A}_{1}$, with a similar central density and mass of the helium shell.

In model $\mathrm{A}_{1}$, the detonation of helium is induced at the edge of the $\mathrm{CO}$ core, at a radius $r=4200 \mathrm{~km}$. Being threedimensional calculations, models $\mathrm{A}_{1}, \mathrm{~B}_{1}, \mathrm{~B}_{2}$, and $\mathrm{B}_{3}$ have a relatively low resolution (see columns 8 and 9 in Table 1). Therefore, to build a sustainable detonation, we artificially incinerate all of the helium fuel inside a sphere with radius $250 \mathrm{~km}$. It takes roughly $0.2 \mathrm{~s}$ to ignition to give rise to a welldefined steady detonation. The duration of the transient stage 


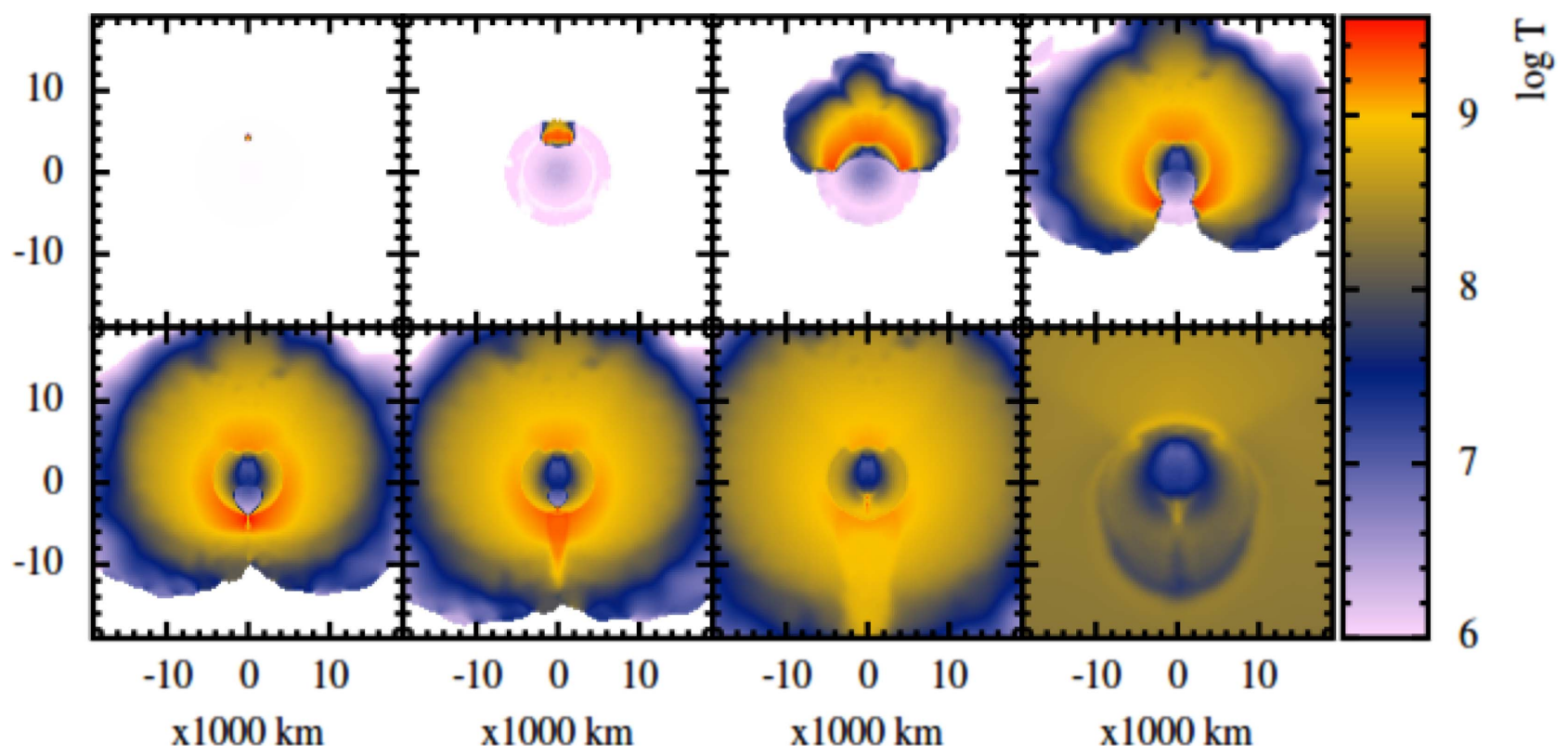

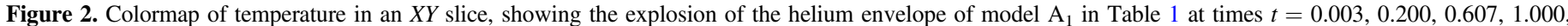

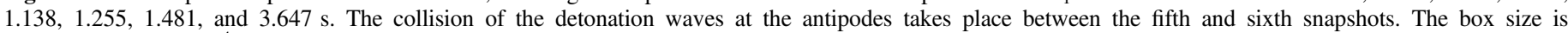
$[-2: 2] \times[-2: 2] \times 10^{4} \mathrm{~km}$.

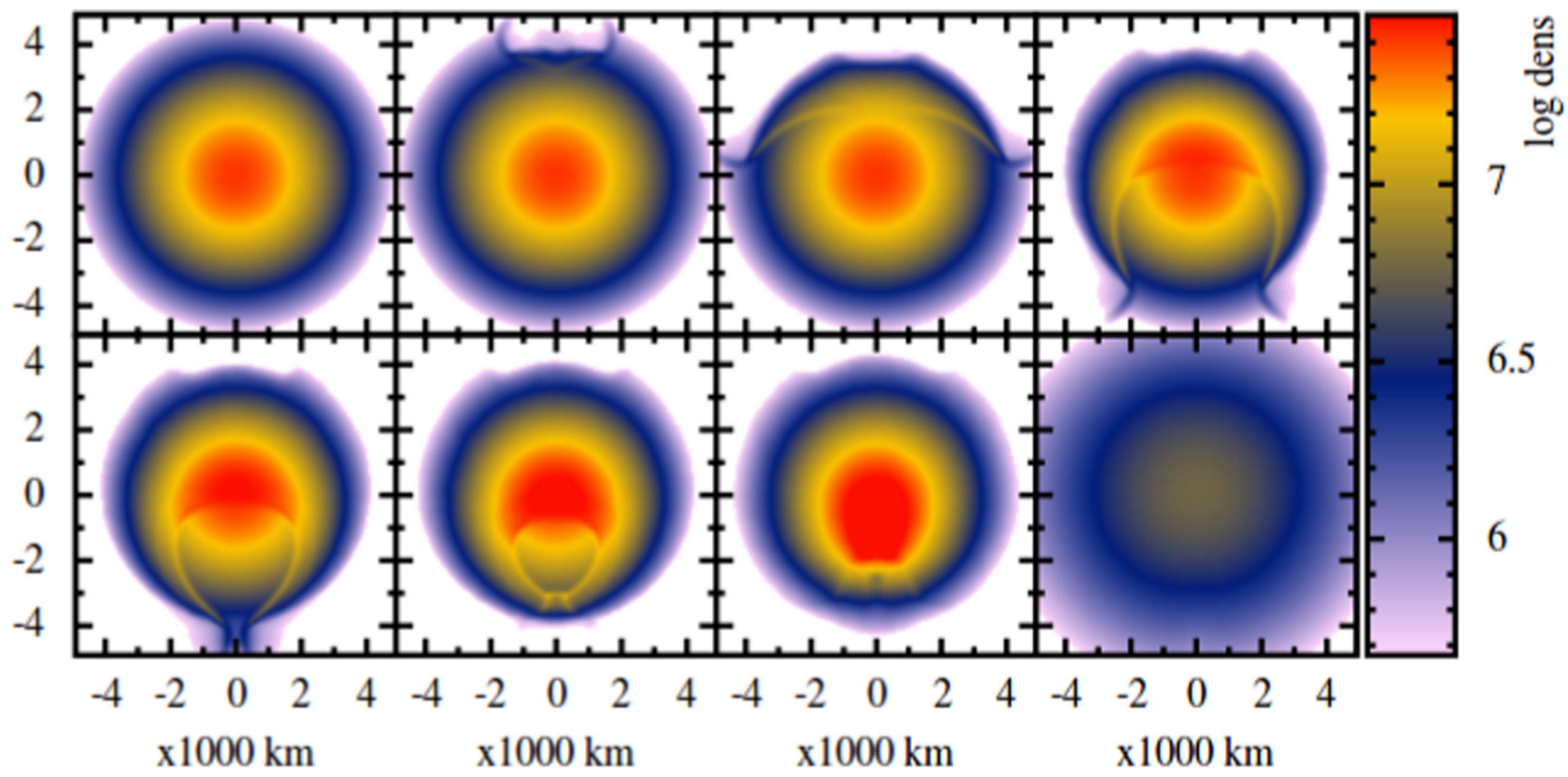

Figure 3. Same as Figure 2 but for density and zoomed in on the central core of the WD. The box size is [-5:5] $\times[-5: 5] \times 10^{3} \mathrm{~km}$.

relies on the resolution and the ignition density. After a while, a steady detonation wave emerges, rapidly incinerating the entire envelope of the WD. The properties and evolution of the $\mathrm{He}$ detonation have been investigated in numerous works in two and three dimensions (Livne \& Arnett 1995; García-Senz et al. 1999; Sim et al. 2010; Moll \& Woosley 2013). On the whole, all of them agree in that the most critical issue is the convergence of the surface detonations at the antipodes of the initial incinerated region. Such convergence is so strong as to induce the detonation of the carbon layer at or below the convergence point. Finally, the detonation of the carbon propagates through the core and volatilizes the star (see Section 5).
The evolution of model $A_{1}$ is in agreement with the findings of previous works. Actually, our results are similar to those of model A by Moll \& Woosley (2013). In Figures 2 and 3, we show the temperature and density colormaps at different times. In this calculation, the combustion of the carbon underneath was turned off to maximize the density achieved during the collision at the antipodes. The convergence of the ashes from the He detonation takes place at $t \simeq 1.18 \mathrm{~s}$, at an altitude of $r \simeq 4000 \mathrm{~km}$. The collision of the ashes raises the temperature and density of carbon to $T \simeq 5.06 \times 10^{9} \mathrm{~K}$ and $\rho \simeq 7.9 \times 10^{6} \mathrm{~g} \mathrm{~cm}^{-3}$, more than enough to initiate the detonation of carbon, if nuclear reactions were switched on (Seitenzahl et al. 2009). In Figure 4, we present the history of the maximum temperature achieved by any particle 

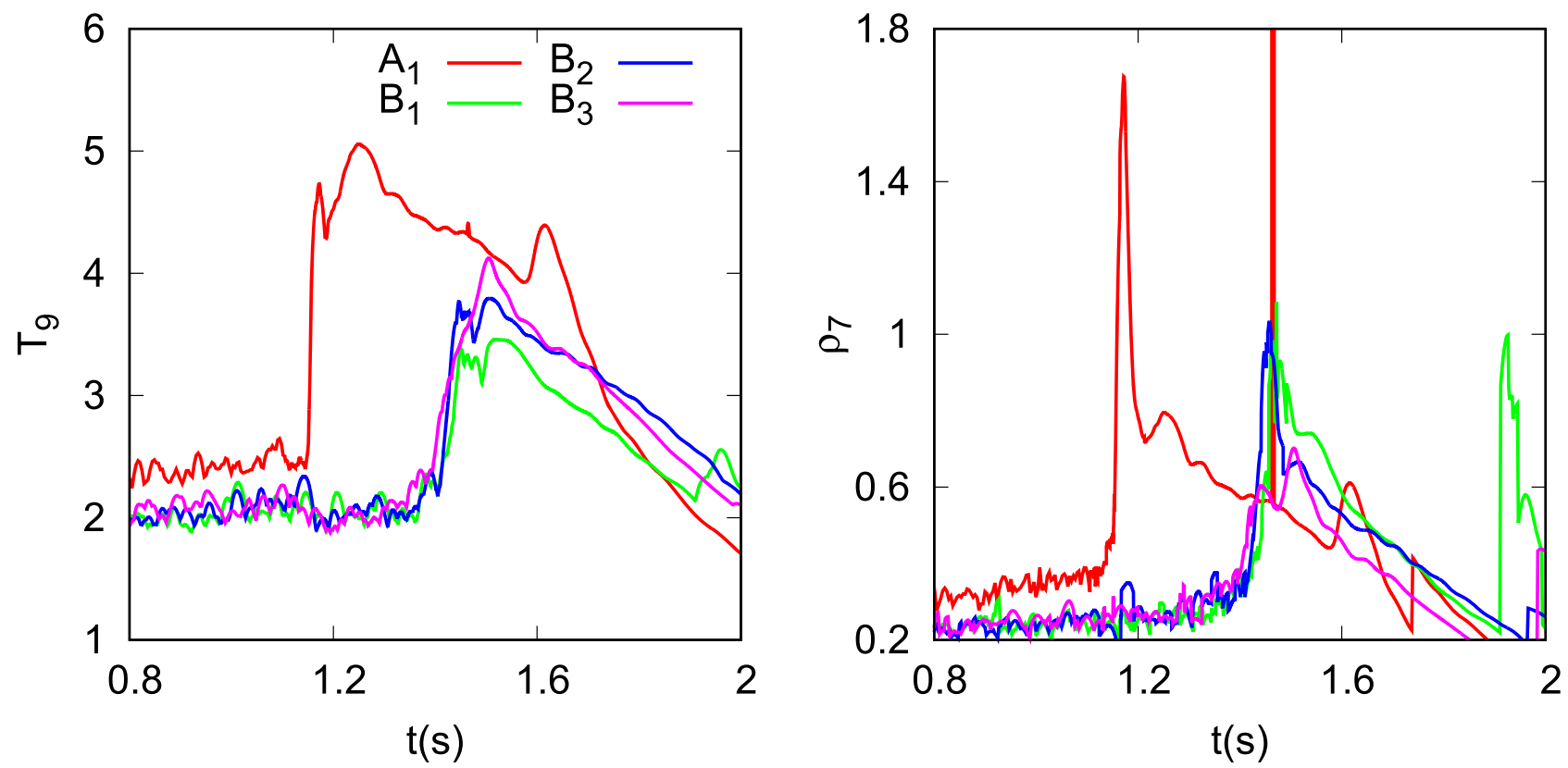

Figure 4. Maximum temperature $T_{\max }$ and its corresponding density $\rho\left(T_{\max }\right)$ for models $\mathrm{A}_{1}, \mathrm{~B}_{1}, \mathrm{~B}_{2}$, and $\mathrm{B}_{3}$. We show here the values achieved by any SPH particle with $\mathrm{CO}$ composition as a function of the elapsed time.

made of carbon and oxygen. The same figure also shows the corresponding density of that particle. As we can see, there is a pronounced plateau in $T_{\max }$ between times $1.16 \leqslant t \leqslant 1.64 \mathrm{~s}$, where $T_{\max } \geqslant 4 \times 10^{9} \mathrm{~K}$, and the $\mathrm{CO}$ mix is prone to detonate. Within this interval, there is a prominent peak in density at $t \simeq$ $1.465 \mathrm{~s}$, where the chances for carbon detonation are maximized. Such high values of density and temperature come after the convergence of the different shock waves at the symmetry axis, at an altitude $r \simeq 1650 \mathrm{~km}$.

The fate of the rotating models may rely on the precise location where the He detonation starts. If the helium detonates just at the rotational axis (model $\mathrm{B}_{1}$ in Table 1), a preferred symmetry line remains, joining the initial ignition spot with the center of the WD, and the evolution should not be very different to that of a spherically symmetric model (i.e., nonrotating). We note, however, that enforcing a similar $\rho_{c}$ and $\Delta M_{\mathrm{He}}$ in rotating and non-rotating models produces slightly different ignition densities of helium at the core edge. As a result, the densities and temperatures in the converging region are higher in the spherically symmetric non-rotating model (Figure 4 and Table 2).

The values of $T_{\max }$ and $\rho\left(T_{\max }\right)$ in the carbon region for rotating B models are shown in Figure 4 (green, blue, and pink lines) and Table 2, respectively. As we can see, the profiles of temperature and density follow a trend similar to that in model $A_{1}$. Nevertheless, the temperature and density peaks in model $\mathrm{B}_{1}$ are less pronounced. They are also delayed approximately $\Delta t \simeq 0.2 \mathrm{~s}$ with respect to model $\mathrm{A}_{1}$. According to the standard detonation criteria (Niemeyer \& Woosley 1997; Seitenzahl et al. 2009), carbon may detonate in model $B_{1}$ when $1.40 \leqslant t \leqslant 1.5 \mathrm{~s}$.

The evolution of models $\mathrm{B}_{2}$ and $\mathrm{B}_{3}$, igniting in a line oblique to the spinning axis, is a bit different. Several snapshots of the explosion of the He layer of model $\mathrm{B}_{3}$ (igniting at the equatorial plane) are depicted in Figures 5-7. The upper row of panels in Figure 5 shows the temperature colormap in an $X Y$ slice containing both the rotational axis and the ignition point
Table 2

Main Features during the Detonation of the He Shell

\begin{tabular}{lccccc}
\hline \hline Model & $\begin{array}{c}\mathrm{T}_{\max } \\
10^{9} \mathrm{~K}\end{array}$ & $\begin{array}{c}\rho\left(T_{\text {max }}\right) \\
10^{7} \mathrm{~g} \mathrm{~cm}^{-3}\end{array}$ & $\begin{array}{c}E_{\text {nuc }} \\
10^{50} \mathrm{erg}\end{array}$ & $\begin{array}{c}{ }^{44} \mathrm{Ti} \\
M_{\odot}\end{array}$ & $\begin{array}{c}{ }^{56} \mathrm{Ni} \\
M_{\odot}\end{array}$ \\
\hline $\mathrm{A}_{1}$ & 5.06 & 0.79 & 1.83 & $2.24 \times 10^{-2}$ & $1.25 \times 10^{-3}$ \\
$\mathrm{~B}_{1}$ & 3.46 & 0.74 & 1.65 & $3.81 \times 10^{-2}$ & $1.18 \times 10^{-4}$ \\
$\mathrm{~B}_{2}$ & 3.79 & 0.66 & 1.66 & $3.79 \times 10^{-2}$ & $1.48 \times 10^{-4}$ \\
$\mathrm{~B}_{3}$ & 4.13 & 0.70 & 1.66 & $3.75 \times 10^{-2}$ & $1.90 \times 10^{-4}$ \\
$\mathrm{~B}_{4}$ & 4.86 & 0.92 & 2.55 & $3.42 \times 10^{-2}$ & $1.24 \times 10^{-3}$ \\
$\mathrm{~B}_{5}$ & 4.50 & 0.95 & 2.56 & $3.41 \times 10^{-2}$ & $1.35 \times 10^{-3}$ \\
$\mathrm{~B}_{6}$ & 3.63 & 0.82 & 2.57 & $3.39 \times 10^{-2}$ & $1.63 \times 10^{-3}$ \\
$\mathrm{C}_{1}$ & 4.54 & 1.36 & 0.88 & $1.33 \times 10^{-2}$ & $2.45 \times 10^{-4}$ \\
$\mathrm{D}_{1}$ & 3.60 & 0.92 & 0.80 & $1.82 \times 10^{-2}$ & $4.67 \times 10^{-5}$ \\
$\mathrm{D}_{2}$ & 3.70 & 0.78 & 0.81 & $1.80 \times 10^{-2}$ & $8.50 \times 10^{-5}$ \\
\hline
\end{tabular}

Note. Columns are the model name, values of $T_{\max }$ and $\rho\left(T_{\max }\right)$, total released nuclear energy, and titanium and nickel abundances exclusively coming from the detonation of the helium shell. The combustion of any particle belonging to the $\mathrm{CO}$ core has been artificially suppressed.

(a polar plane). Such a polar plane is rotating with $\omega_{x}=0.5 \mathrm{~s}^{-1}$, so that it is a comoving projection plane. On the other hand, the lower row in the same figure shows the temperature in the equatorial plane as viewed from a non-rotating frame of reference. On the whole, the geometry of the oblate spheroid desynchronizes the convergence of the ashes at the antipodes. This is more evident in the colormap of density, Figure 6, and especially in the close-up of Figure 7, which focuses on the convergence region. As we can see, convergence is attained earlier in the polar plane than in the equatorial plane. Such a shift in the converging times is purely geometric, because in an oblate spheroid, the polar geodesic has a length $l_{\mathrm{pol}}=2 \pi a$, whereas the equatorial geodesic amounts to $l_{\mathrm{eq}}=2 \pi \sqrt{0.5\left(a^{2}+b^{2}\right)}$, where $a$ and $b$ are the equatorial and the polar radius. According to the values in Table $1, l_{\text {eq }} / l_{\text {pol }} \simeq 1.18$; any other geodesic has $l_{g}$ with $l_{\mathrm{pol}} \leqslant l_{g} \leqslant l_{\text {eq }}$. Admitting an isotropic distribution of detonation velocities, there is a continuous shift in the arrival times of 


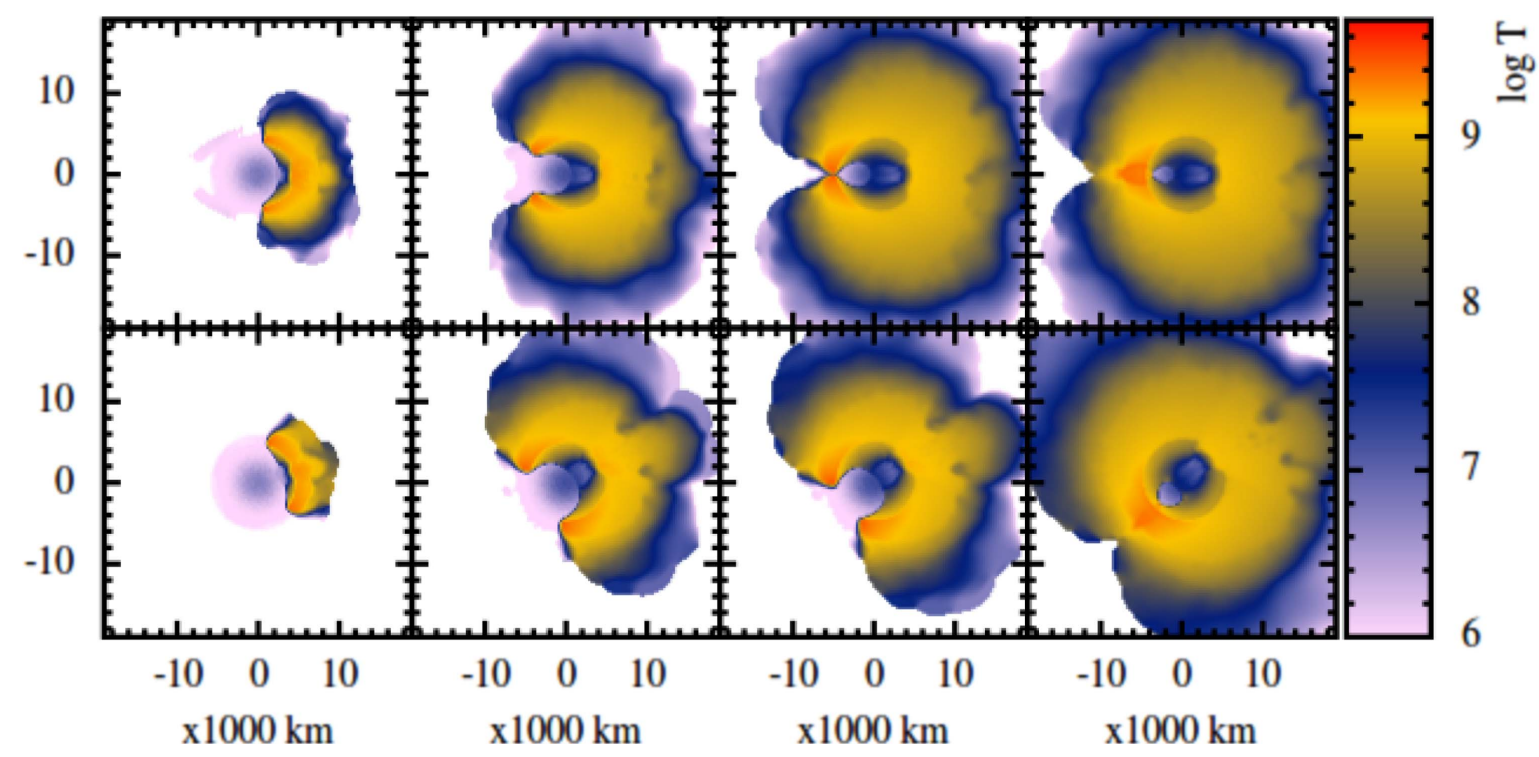

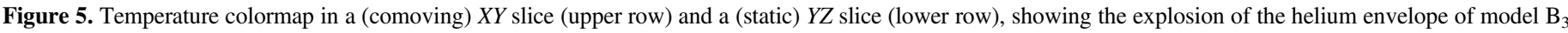

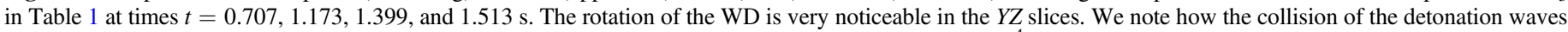
at the antipodes takes place at quite different times in both slice sequences. The box size is $[-2: 2] \times 10^{4} \mathrm{~km}$ in all directions.

the converging waves. Therefore, the strong focusing that characterizes models $A_{1}$ and $B_{1}$ is somehow lost in models $B_{2}$ and, more evidently, $\mathrm{B}_{3}$. Still, the wave convergence at the antipodes is strong enough as to induce the detonation of carbon. Just imagine the picture from a rotating reference frame: as the detonation is supersonic, the forces acting on a fluid element that goes through the shock front are much higher than the noninertial-centripetal and Coriolis-forces, which do not appreciably affect the propagation of the detonation wave. ${ }^{6}$

The principal impact of rotation is to desynchronize the wave trains arriving at the antipodes of the ignition region. Such asynchronous wave arrival, however, does not necessarily reduce the peak temperature deep down the antipodes. According to Figure 4 and Table 2, the largest value of $T_{\max }$ for rotating models is actually achieved in model $\mathrm{B}_{3}\left(T_{\max }=4.13 \times 10^{9} \mathrm{~K}\right)$, followed by $\mathrm{B}_{2}\left(3.79 \times 10^{9} \mathrm{~K}\right)$ and $\mathrm{B}_{1}\left(3.46 \times 10^{9} \mathrm{~K}\right)$, with densities $\rho \simeq 0.7 \times 10^{7} \mathrm{~g} \mathrm{~cm}^{-3}$ in all three cases. If the ${ }^{12} \mathrm{C}+$ ${ }^{12} \mathrm{C}$ reaction had been switched on, these temperatures and densities would have been high enough (Niemeyer \& Woosley 1997; Seitenzahl et al. 2009) to provoke the detonation of the core of the WD (see Section 5). We conclude that the ignition and detonation of carbon are the most probable outcomes in all rotating models that we calculated. Therefore, the DDet mechanism appears to be robust: it not only works if helium is ignited in one or several points (García-Senz et al. 1999; Moll \& Woosley 2013) but also when the WD is rapidly rotating.

The yields produced during the detonation of the helium shell are shown in Table 3 . These yields are only approximate owing to the small size of the 14 nuclei network used to track the He detonation. The main limitation comes, however, from the low resolution achieved in the helium envelope, which results in a large fraction of unburned helium after the freezing of the reactions at $t \geqslant 2 \mathrm{~s}$. This is because of the limited capabilities of SPH codes to handle shock waves moving in low-density regions. In diluted regions, the smoothing length gets larger, and

\footnotetext{
6 Nevertheless, the inertial forces have some impact on the large-scale geometry of the explosions. In particular, the centrifugal barrier set by the rotation favors elongated morphologies along the rotational axis (Pfannes et al. 2010a, 2010b).
}

the shock wave front is strongly smeared, making it more difficult for the detonation to propagate outwards in the envelope. Compared to the spherically symmetric model $\mathrm{A}_{1}$, the final abundance of ${ }^{56} \mathrm{Ni}$ is approximately an order of magnitude lower in B models. The higher production of nickel in the non-rotating model is due to (a) the slightly higher ignition density of helium in model $\mathrm{A}_{1}$, (b) the higher densities and temperatures achieved at the converging region in model $\mathrm{A}_{1}$ (Figure 4), and (c) fast rotators having a larger amount of mass "stored" at low densities, which disfavors the production of iron-group elements (IGE). In all cases, but especially in the rotating models, the more abundant ejected species are the radioactive ${ }^{44} \mathrm{Ti}$ and ${ }^{48} \mathrm{Cr}$. We note that the presence of $\mathrm{Ti}$ absorption lines in the near maximum spectra has been suggested as an indicator of the He-detonation-triggered scenario (Jiang et al. 2017).

As pointed out in previous works by other authors (Sim et al. 2012), the detonation of the He shell alone would produce a subluminous event $\left(M_{\text {bol }} \simeq-16.5\right)$ with a peculiar light curve dominated by the disintegration of ${ }^{52} \mathrm{Fe}$ rather than ${ }^{56} \mathrm{Ni}$ at early times.

\subsection{Geometry of the Ejected Shell}

A point-like, edge-lit ignition of the helium envelope, whether or not followed by the complete detonation of the CO core of the WD, leads to a loss of spherical symmetry, which may be detected in polarization studies (Fink et al. 2010; Bulla et al. 2016; Bulla 2017). We want to investigate if such a loss of spherical symmetry is more pronounced in rotating WDs. In Figure 8, we show the combined column density of radioactive ${ }^{48} \mathrm{Cr}+{ }^{52} \mathrm{Fe}+{ }^{56} \mathrm{Ni}$ for different models, at $t \simeq 8.3 \mathrm{~s}$, when the expansion is homologous. Such a column density ${ }^{7}$ is estimated by assuming an artificial photosphere with local thickness $2 \bar{h}$ (being $\bar{h}$ the average of the smoothing length) and projected onto three orthogonal observer planes, $Y Z, X Y, X Z$ (with the plane $Y Z$ parallel to the equator of the WD). Because these radioactive elements are expanding

Obtained and drawn with the public program SPLASH written by D. Price (2007). 


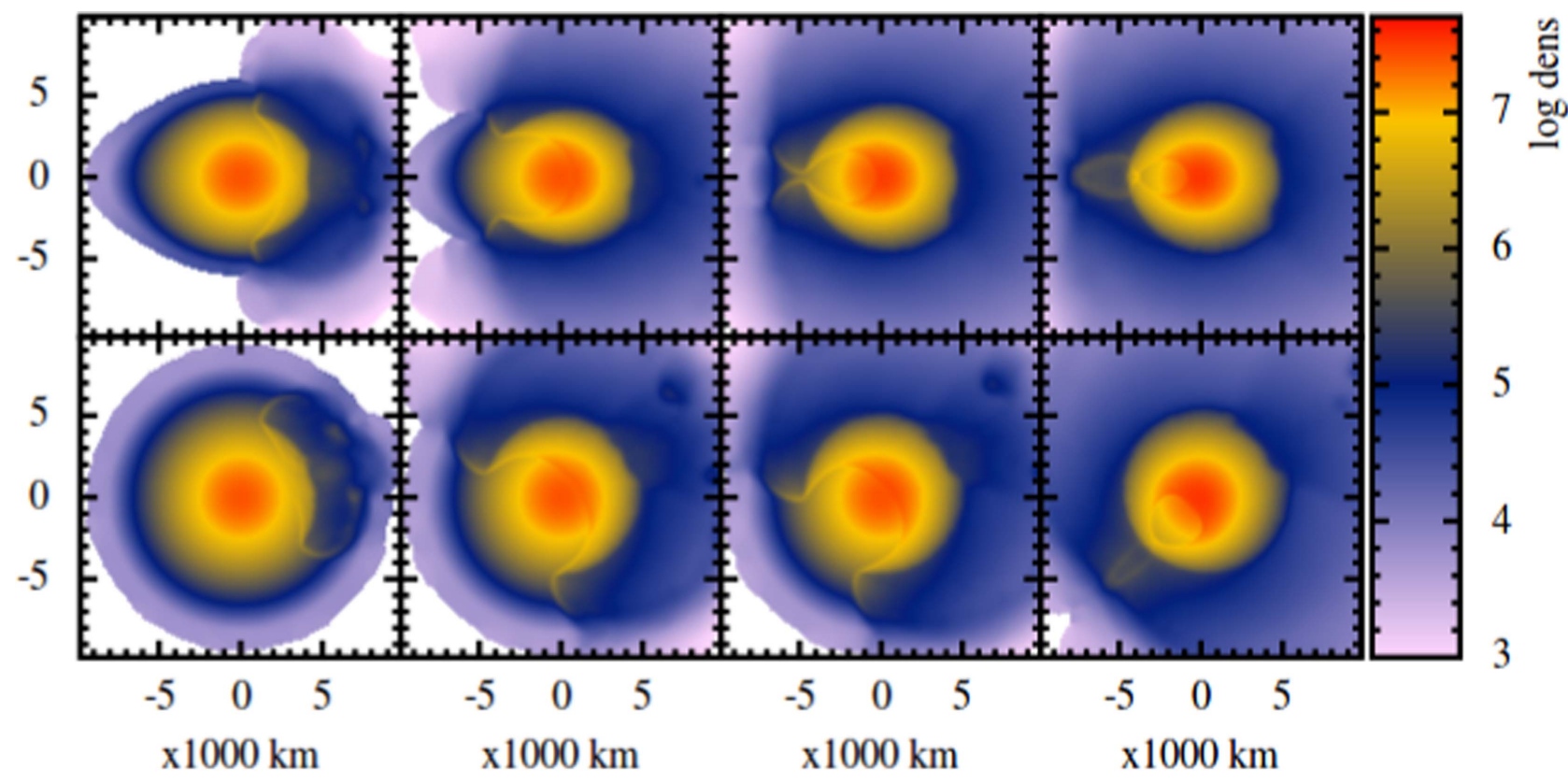

Figure 6. Same as Figure 5, but for density. The box size is $[-1: 1] \times 10^{4} \mathrm{~km}$ in all directions.

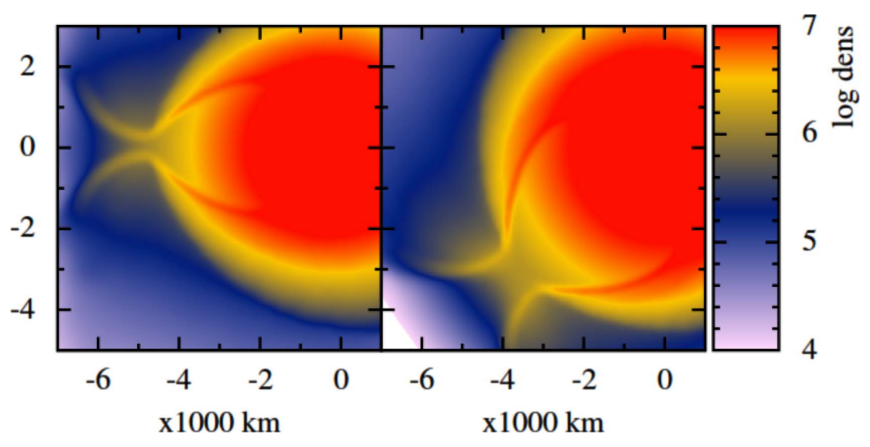

Figure 7. Colormap of density around the convergence region at time $t=1.399 \mathrm{~s}$ showing the time shift among wave arrivals in the polar plane $X Y$ (left) and equatorial $Y Z$ (right).

homologously, their relative spatial distribution will not change afterwards with time after $t \simeq 8.5 \mathrm{~s}$, up to the moment at which these elements begin to disintegrate several days after.

The upper row of panels in Figure 8 depicts the geometry of the artificial photosphere for the non-rotating model $\mathrm{A}_{1}$. The distribution of radioactive ${ }^{48} \mathrm{Cr}+{ }^{52} \mathrm{Fe}+{ }^{56} \mathrm{Ni}$ is not totally spherical when viewed perpendicularly to the polar direction (central and rightmost snapshots), with a larger concentration in the northern hemisphere. On another note, the distribution is rather smooth, free from pockets of ${ }^{56} \mathrm{Ni}$, which characterize pure deflagration models (García-Senz \& Bravo 2005). The impact of such an asymmetric distribution of IGEs and intermediate-mass elements (IMEs) in the polarization of the spectra in subCh-mass models has been recently analyzed by Bulla et al. (2016). They conclude that the asymmetries are not large enough to produce significant levels of polarization $(\geqslant 0.5 \%)$ in the spectra. We note that the polar view (leftmost snapshot) is totally symmetric, as expected.

Figure 8 also shows the column density of the radioactive elements synthesized during the He detonation of rotating models $\mathrm{B}_{1}, \mathrm{~B}_{2}$, and $\mathrm{B}_{3}$. In particular, models $\mathrm{B}_{1}$ and $\mathrm{B}_{2}$ look similar to the control model $\mathrm{A}_{1}$, but they are slightly more elongated in the direction of the rotational axis (central and rightmost columns in
Figure 8). Such anisotropic distribution of the burning products is due to the angular momentum barrier set by the rotation, which is stronger in the equatorial direction (Pfannes et al. 2010b). Interestingly, the distribution of radioactive elements in model $\mathrm{B}_{3}$ seems to be more spherical than in models $\mathrm{B}_{1}$ and $\mathrm{B}_{2}$ in those planes. When viewed from the polar axis (leftmost column in Figure 8 ), models $B_{1}$ and $B_{2}$ look similar to $A_{1}$, but $B_{3}$ has a clear loss of spherical symmetry. Although the loss of spherical symmetry is larger than in the non-rotating model, providing quantitative numbers for its impact on the polarization of the spectra is beyond the scope of the present work.

To sum up, the single detonation of the helium shell in a rotating $\simeq 1 M_{\odot}$ WD would produce a subluminous event powered by the disintegration of ${ }^{48} \mathrm{Cr}+{ }^{52} \mathrm{Fe}$ and ${ }^{56} \mathrm{Ni}$. The asymmetries in the distribution of nuclear species are larger than in spherically symmetric models, which probably will increase the level of polarization in the light curve and spectra. It is worth noting that our model $A_{1}$ does not give yields of ${ }^{48} \mathrm{Cr}$ very different from those obtained by Sim et al. (2012) for an explosion model igniting helium at a slightly lower density (their model HeD-S). Nevertheless, model $\mathrm{A}_{1}$ synthesizes more ${ }^{44} \mathrm{Ti}$, with a ${ }^{44} \mathrm{Ti} /{ }^{48} \mathrm{Cr}$ ratio a factor of $\simeq 2$ larger than that in Sim et al. (2012). Such a discrepancy may come from the different initial models, hydrodynamic method, 2D (Sim et al.) versus 3D, and nuclear treatment. On another note, the half-life of ${ }^{44} \mathrm{Ti}$ ( $\simeq 60$ years $)$ is quite large, thus it does not leave a sizable fingerprint in the light curve profile during the first months after the explosion.

The detonation of the He shell alone would produce, if observed, an intermediate event between a nova explosion and an SN Ia. As hydrogen is absent in the spectra and there is some ${ }^{28} \mathrm{Si}$, it ought to be classified as Type Ia, but as a subluminous peculiar one. In order to produce an amount of ${ }^{56} \mathrm{Ni}$ compatible with what is observed in a standard SN Ia explosion, it is also necessary to obtain the detonation of the $\mathrm{CO}$ core. According to our results (see Figure 4), core detonation is also the most probable outcome, even when the WD rotates fast, close to the centrifugal breaking. 
Table 3

Yields Synthesized during the Combustion of the He Shell (in $M_{\odot}$ )

\begin{tabular}{|c|c|c|c|c|c|c|c|c|c|c|}
\hline & $\overline{A_{1}}$ & $\mathrm{~B}_{1}$ & $\mathrm{~B}_{2}$ & $\mathrm{~B}_{3}$ & $\mathrm{~B}_{4}$ & $\mathrm{~B}_{5}$ & $\mathrm{~B}_{6}$ & $\mathrm{C}_{1}$ & $\mathrm{D}_{1}$ & $\mathrm{D}_{2}$ \\
\hline${ }^{4} \mathrm{He}$ & $4.20 \times 10^{-2}$ & $5.47 \times 10^{-2}$ & $5.45 \times 10^{-2}$ & $5.43 \times 10^{-2}$ & $6.31 \times 10^{-2}$ & $6.28 \times 10^{-2}$ & $6.23 \times 10^{-2}$ & $2.06 \times 10^{-2}$ & $2.44 \times 10^{-2}$ & $2.40 \times 10^{-2}$ \\
\hline${ }^{12} \mathrm{C}$ & $1.92 \times 10^{-4}$ & $4.51 \times 10^{-4}$ & $4.34 \times 10^{-4}$ & $4.43 \times 10^{-4}$ & $2.99 \times 10^{-4}$ & $2.98 \times 10^{-4}$ & $3.01 \times 10^{-4}$ & $1.70 \times 10^{-4}$ & $3.25 \times 10^{-4}$ & $2.95 \times 10^{-4}$ \\
\hline${ }^{16} \mathrm{O}$ & $5.35 \times 10^{-7}$ & $8.67 \times 10^{-7}$ & $8.57 \times 10^{-7}$ & $8.57 \times 10^{-7}$ & $7.88 \times 10^{-7}$ & $7.80 \times 10^{-7}$ & $7.92 \times 10^{-7}$ & $3.47 \times 10^{-7}$ & $5.03 \times 10^{-7}$ & $4.83 \times 10^{-7}$ \\
\hline${ }^{20} \mathrm{Ne}$ & $6.05 \times 10^{-8}$ & $1.24 \times 10^{-7}$ & $1.18 \times 10^{-7}$ & $1.20 \times 10^{-7}$ & $9.22 \times 10^{-8}$ & $9.10 \times 10^{-8}$ & $9.15 \times 10^{-8}$ & $4.64 \times 10^{-8}$ & $8.40 \times 10^{-8}$ & $7.50 \times 10^{-8}$ \\
\hline${ }^{24} \mathrm{Mg}$ & $4.77 \times 10^{-7}$ & $1.14 \times 10^{-6}$ & $1.07 \times 10^{-6}$ & $1.09 \times 10^{-6}$ & $7.44 \times 10^{-7}$ & $7.34 \times 10^{-7}$ & $7.33 \times 10^{-7}$ & $4.05 \times 10^{-7}$ & $8.41 \times 10^{-7}$ & $7.17 \times 10^{-7}$ \\
\hline${ }^{28} \mathrm{Si}$ & $5.02 \times 10^{-6}$ & $1.25 \times 10^{-5}$ & $1.22 \times 10^{-5}$ & $1.23 \times 10^{-5}$ & $7.95 \times 10^{-6}$ & $7.97 \times 10^{-6}$ & $8.20 \times 10^{-6}$ & $4.73 \times 10^{-6}$ & $9.24 \times 10^{-6}$ & $8.59 \times 10^{-6}$ \\
\hline${ }^{32} \mathrm{~S}$ & $5.96 \times 10^{-5}$ & $1.26 \times 10^{-4}$ & $1.25 \times 10^{-4}$ & $1.27 \times 10^{-4}$ & $9.26 \times 10^{-5}$ & $9.25 \times 10^{-5}$ & $9.80 \times 10^{-5}$ & $5.32 \times 10^{-5}$ & $8.35 \times 10^{-5}$ & $8.27 \times 10^{-5}$ \\
\hline${ }^{36} \mathrm{Ar}$ & $9.74 \times 10^{-4}$ & $1.56 \times 10^{-3}$ & $1.55 \times 10^{-3}$ & $1.52 \times 10^{-3}$ & $1.56 \times 10^{-3}$ & $1.55 \times 10^{-3}$ & $1.55 \times 10^{-3}$ & $6.43 \times 10^{-4}$ & $8.06 \times 10^{-4}$ & $7.72 \times 10^{-4}$ \\
\hline${ }^{40} \mathrm{Ca}$ & $4.73 \times 10^{-4}$ & $7.22 \times 10^{-4}$ & $7.16 \times 10^{-4}$ & $7.01 \times 10^{-4}$ & $7.72 \times 10^{-4}$ & $7.72 \times 10^{-4}$ & $7.62 \times 10^{-4}$ & $2.89 \times 10^{-4}$ & $3.50 \times 10^{-4}$ & $3.34 \times 10^{-4}$ \\
\hline${ }^{44} \mathrm{Ti}$ & $2.24 \times 10^{-2}$ & $3.81 \times 10^{-2}$ & $3.79 \times 10^{-2}$ & $3.75 \times 10^{-2}$ & $3.43 \times 10^{-2}$ & $3.41 \times 10^{-2}$ & $3.39 \times 10^{-2}$ & $1.33 \times 10^{-2}$ & $1.82 \times 10^{-2}$ & $1.80 \times 10^{-2}$ \\
\hline${ }^{48} \mathrm{Cr}$ & $3.07 \times 10^{-2}$ & $1.70 \times 10^{-2}$ & $1.74 \times 10^{-2}$ & $1.78 \times 10^{-2}$ & $4.15 \times 10^{-2}$ & $4.16 \times 10^{-2}$ & $4.15 \times 10^{-2}$ & $1.41 \times 10^{-2}$ & $8.43 \times 10^{-3}$ & $8.92 \times 10^{-3}$ \\
\hline${ }^{52} \mathrm{Fe}$ & $8.67 \times 10^{-3}$ & $1.06 \times 10^{-3}$ & $1.12 \times 10^{-3}$ & $1.28 \times 10^{-3}$ & $1.05 \times 10^{-2}$ & $1.07 \times 10^{-2}$ & $1.12 \times 10^{-2}$ & $2.45 \times 10^{-3}$ & $5.60 \times 10^{-4}$ & $6.32 \times 10^{-4}$ \\
\hline${ }^{56} \mathrm{Ni}$ & $1.25 \times 10^{-3}$ & $1.18 \times 10^{-4}$ & $1.48 \times 10^{-4}$ & $1.90 \times 10^{-4}$ & $1.25 \times 10^{-3}$ & $1.35 \times 10^{-3}$ & $1.63 \times 10^{-3}$ & $2.45 \times 10^{-4}$ & $4.67 \times 10^{-5}$ & $8.50 \times 10^{-5}$ \\
\hline${ }^{60} \mathrm{Zn}$ & $1.83 \times 10^{-5}$ & $1.39 \times 10^{-6}$ & $1.63 \times 10^{-6}$ & $2.42 \times 10^{-6}$ & $1.94 \times 10^{-5}$ & $2.11 \times 10^{-5}$ & $2.60 \times 10^{-5}$ & $3.24 \times 10^{-6}$ & $5.70 \times 10^{-7}$ & $9.65 \times 10^{-7}$ \\
\hline
\end{tabular}




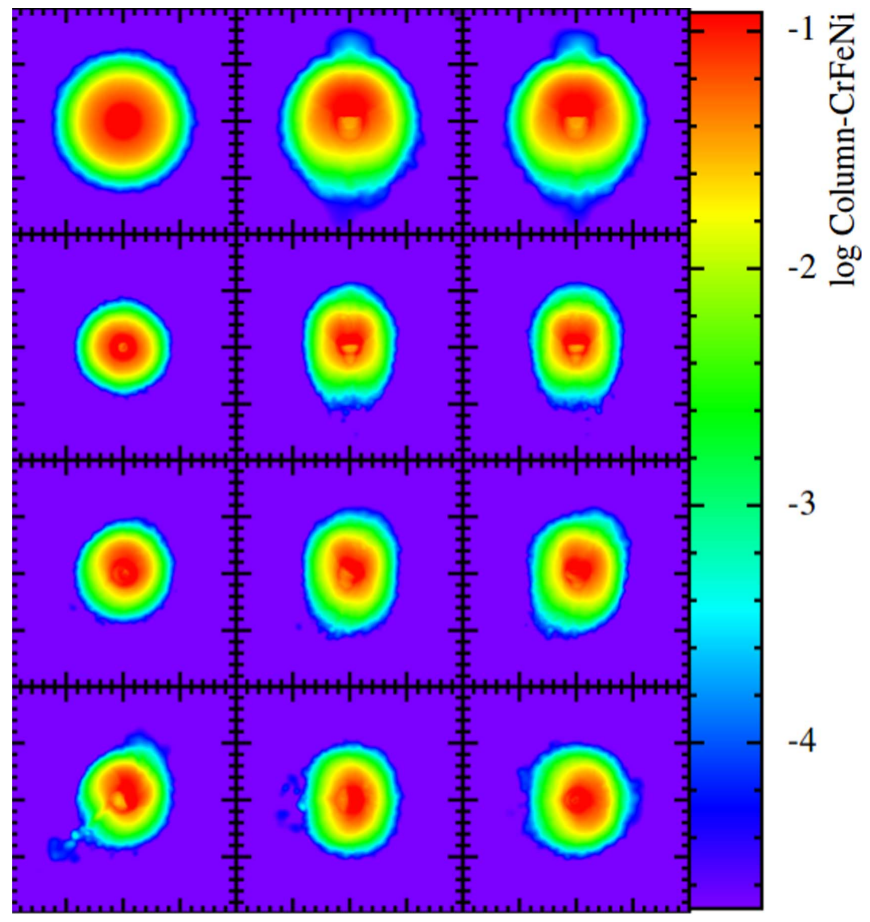

Figure 8. From left to right: column densities of the radioactive ${ }^{48} \mathrm{Cr}+{ }^{52} \mathrm{Fe}+$ ${ }^{56} \mathrm{Ni}$ mass fractions along the $X$ (polar view), $Y$, and $Z$ directions at times $t \simeq 8.57,8.07,8.32$, and $8.12 \mathrm{~s}$ for models $\mathrm{A}_{1}, \mathrm{~B}_{1}, \mathrm{~B}_{2}$, and $\mathrm{B}_{3}$ (from top to bottom), respectively. The boxes have a side length of $4 \times 10^{5} \mathrm{~km}$.

\subsection{He-shell Detonation: Increasing the Ignition Density at the Core-Envelope Interface}

The precise value at which the first sparks of helium ignite has a strong impact on some of the yields coming from the detonation of the helium shell. The reference models $B_{1}$, $\mathrm{B}_{2}$, and $\mathrm{B}_{3}$ discussed above assumed a low ignition density value, $\rho_{\mathrm{He}}=1.1 \times 10^{6} \mathrm{~g} \mathrm{~cm}^{-3}$, close to the minimum necessary to build a steady detonation. The impact of raising the ignition density of helium at the interface up to $\rho_{\mathrm{He}}=$ $1.5 \times 10^{6} \mathrm{~g} \mathrm{~cm}^{-3}$ is explored in models $\mathrm{B}_{4}, \mathrm{~B}_{5}$, and $\mathrm{B}_{6}$. As the base of the He shell is moved deeper, its mass and thickness increase so that the total mass of the WD remains constant (see Table 1). The combination of a higher ignition density and a more massive envelope (i.e., a larger explosion tamper) leads to higher combustion temperatures, thus favoring the synthesis of IGEs. In particular, the ${ }^{52} \mathrm{Fe}$ and ${ }^{56} \mathrm{Ni}$ yields are increased by a factor of 10 (Table 2) while the released nuclear energy rises by $50 \%$ (Table 4). The largest amount of $\mathrm{Fe}-\mathrm{Ni}$ is synthesized in the off-axis igniter $\mathrm{B}_{6}$, whereas the aligned igniter, model $\mathrm{B}_{4}$, gives an amount of IGE similar to those of the nonrotating model $\mathrm{A}_{1}$.

The evolution of $T_{\max }$ (maximum temperature in the core with the nuclear reactions turned off) and $\rho\left(T_{\max }\right)$ of models $\mathrm{B}_{4}, \mathrm{~B}_{5}$, and $\mathrm{B}_{6}$ is shown in Figure 9. The maximum temperature and densities achieved in models $\mathrm{B}_{4}$ and $\mathrm{B}_{5}$ are larger than those in models $\mathrm{B}_{1}$ and $\mathrm{B}_{2}$. Even though model $\mathrm{B}_{6}$ has a peak $T_{\max }$ similar to that of $\mathrm{B}_{3}$, the evolution of $\rho\left(T_{\max }\right)$ is quite different because it has an extended plateau where $\rho\left(T_{\max }\right) \simeq 10^{7}$ $\mathrm{g} \mathrm{cm}^{-3}$ between 1.4 and $1.7 \mathrm{~s}$. Therefore, the conditions to induce the detonation of the core are even more favorable in models $\mathrm{B}_{4}, \mathrm{~B}_{5}$, and $\mathrm{B}_{6}$ than in models $\mathrm{B}_{1}, \mathrm{~B}_{2}$, and $\mathrm{B}_{3}$, which ignite helium at a lower density at the interface.
Table 4

Main Features during the Complete Detonation of the WD

\begin{tabular}{lccccc}
\hline \hline Model & $\begin{array}{c}E_{\text {kin }} \\
10^{51} \text { erg }\end{array}$ & $\begin{array}{c}E_{\text {nuc }} \\
10^{51} \text { erg }\end{array}$ & $\begin{array}{c}\text { IME } \\
M_{\odot}\end{array}$ & $\begin{array}{c}\text { IGE } \\
M_{\odot}\end{array}$ & $\begin{array}{c}{ }^{56} \mathrm{Ni} \\
M_{\odot}\end{array}$ \\
\hline $\mathrm{A}_{1}$ & 1.09 & 1.22 & 0.36 & 0.45 & 0.37 \\
$\mathrm{~B}_{4}$ & 1.24 & 1.40 & 0.38 & 0.52 & 0.42 \\
$\mathrm{~B}_{5}$ & 1.27 & 1.43 & 0.38 & 0.54 & 0.44 \\
$\mathrm{~B}_{6}$ & 1.26 & 1.41 & 0.38 & 0.53 & 0.43 \\
$\mathrm{C}_{1}$ & 1.40 & 1.55 & 0.23 & 0.79 & 0.74 \\
$\mathrm{D}_{2}$ & 1.48 & 1.66 & 0.26 & 0.83 & 0.78 \\
\hline
\end{tabular}

Note. Main features of the complete detonation of models $A_{1}, B_{4}, B_{5}, B_{6}, C_{1}$, and $\mathrm{D}_{2}$ at $t=11.5 \mathrm{~s}$.

\subsection{Models with a Thinner He Layer}

One historical objection to the subCh-mass route to SNe Ia is that it predicts a too large nickel production in the high-velocity external layers, which is not seen in the spectra. As suggested by Bildsten et al. (2007), one remedy is to consider thinner helium envelopes so that the amount of synthesized ${ }^{56} \mathrm{Ni}$ is proportionally reduced. But this poses a problem to the robustness of the DDet mechanism, as it may not work below some critical mass of the envelope. Nevertheless, several multidimensional studies have shown that the DDet mechanism may work even for envelopes as small as $\Delta \mathrm{He} \simeq 0.01 M_{\odot}$ (Fink et al. 2010; Sim et al. 2012). It is worth noting that SNe Ia may also arise from the violent merger of two massive CO-WDs capped with tiny helium shells, $\simeq 0.005 M_{\odot}$ each (Guillochon et al. 2010; Pakmor et al. 2013). Hydrodynamic simulations by Pakmor et al. (2013) predict that $\mathrm{He}$ detonation may induce the detonation of the assumed non-rotating, $\mathrm{CO}$ core. Thus, the explosion mechanism invoked in this DD model is rather similar to the DDet mechanism on rotating WDs presented in this work.

We have studied three additional cases, namely $\mathrm{C}_{1}, \mathrm{D}_{1}$, and $\mathrm{D}_{2}$ in Table 1, with $\Delta M_{\mathrm{He}} \simeq 0.05 M_{\odot}$, which is half of the Heenvelope mass used in the $\mathrm{B}$ models. Model $\mathrm{C}_{1}$ is the new nonrotating control case with spherical symmetry, central density $\rho_{c}=6.82 \times 10^{7} \mathrm{~g} \mathrm{~cm}^{-3}$, and $\Delta M_{\mathrm{He}}=0.052 M_{\odot}$. The rotating models, $\mathrm{D}_{1}$ and $\mathrm{D}_{2}$, spin with $w=0.65 \mathrm{~s}^{-1}$ and have a central density $\rho_{c}=6.87 \times 10^{7} \mathrm{~g} \mathrm{~cm}^{-3}$ and $\Delta M_{\mathrm{He}}=0.053 M_{\odot}$. In spite of having a larger rotational velocity, D models are not as oblate as $\mathrm{B}$ models because they are more massive (see Table 1).

The evolution of cases $C_{1}, D_{1}$, and $D_{2}$ is similar to that of models with thicker helium envelopes. Table 2 presents a summary of the results. Again, the maximum temperature $T_{\max }$ and $\rho\left(T_{\max }\right)$ (estimated with the ${ }^{12} \mathrm{C}+{ }^{12} \mathrm{C}$ reaction turned off) achieved by a carbon particle at the antipodes is high enough to induce the detonation of the core. If the density at the edge of the core is similar for all models, then the energy released during the evaporation of the helium envelope roughly scales with the mass of the He shell (Table 2)

\section{Hydrodynamic Simulations: The Core Detonation}

Now we compute models $\mathrm{A}_{1}, \mathrm{~B}_{4}, \mathrm{~B}_{5}, \mathrm{~B}_{6}, \mathrm{C} 1$, and $\mathrm{D}_{2}$ in Table 1 , allowing the binary ${ }^{12} \mathrm{C}+{ }^{12} \mathrm{C},{ }^{12} \mathrm{C}+{ }^{16} \mathrm{O}$, and ${ }^{16} \mathrm{O}+{ }^{16} \mathrm{O}$ reactions to proceed. In all cases, the spontaneous detonation of the core and the complete destruction of the WD are obtained. The released nuclear energy, final kinetic energy, and the rough nucleosynthesis do match the most basic $\mathrm{SNe}$ Ia observational constraints. A summary of these magnitudes is provided in Table 4. Models $\mathrm{A}_{1}$ and $\mathrm{B}_{4,5,6}$ were chosen as representative of explosions born in thick helium shells, whereas 

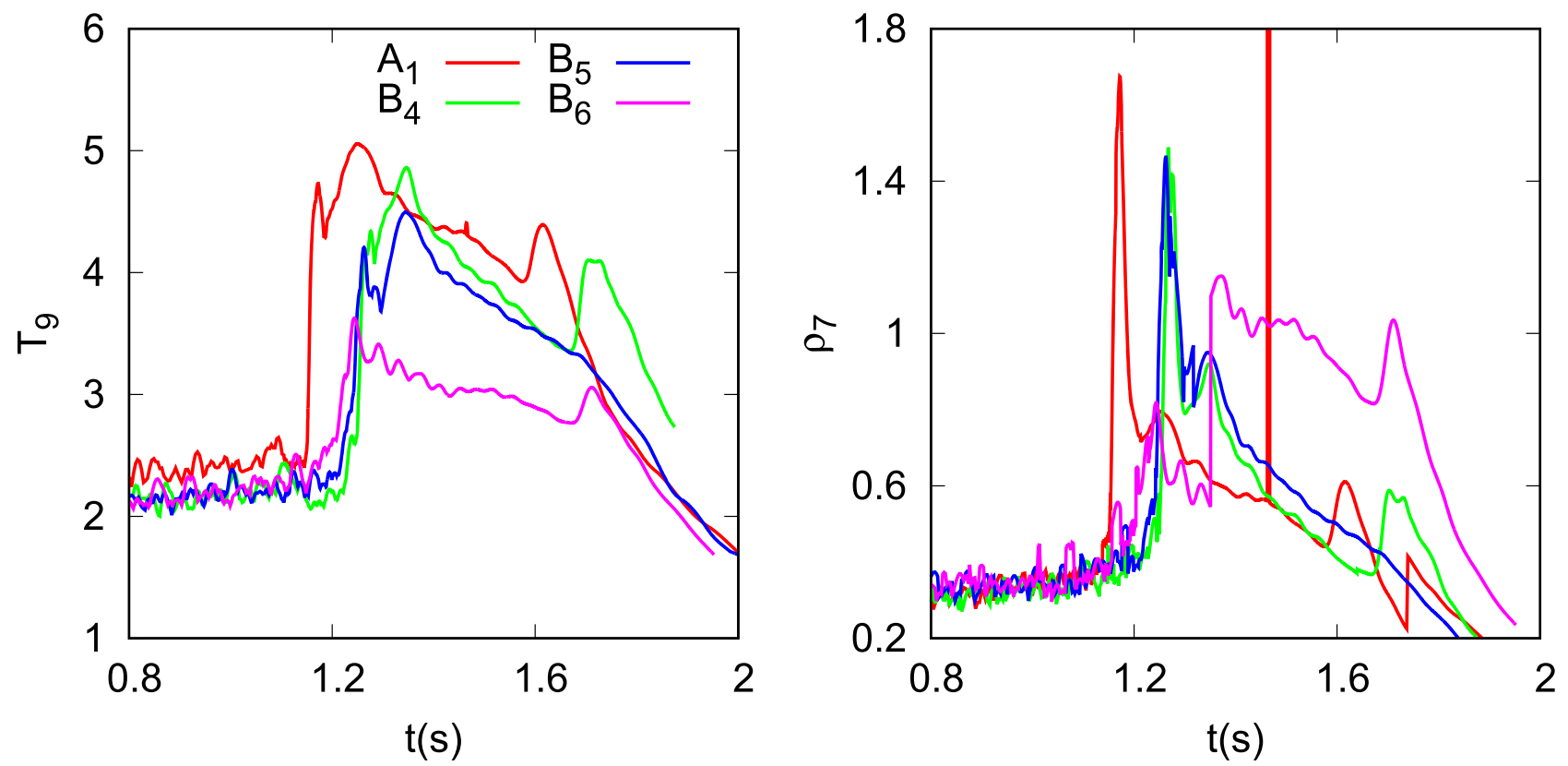

Figure 9. Maximum temperature $T_{\max }$ and its corresponding density $\rho\left(T_{\max }\right)$ for models $\mathrm{A}_{1}, \mathrm{~B}_{4}, \mathrm{~B}_{5}$, and $\mathrm{B}_{6}$. As in Figure 4 , we show here the values achieved by any SPH particle with $\mathrm{CO}$ composition as a function of the elapsed time.

Table 5

Yields Synthesized during the Complete Detonation of the WD (in $M_{\odot}$ )

\begin{tabular}{lccccc}
\hline \hline & $\mathrm{A}_{1}$ & $\mathrm{~B}_{4}$ & $\mathrm{~B}_{5}$ & $\mathrm{~B}_{6}$ & $\mathrm{C}_{1}$ \\
\hline${ }^{4} \mathrm{He}$ & $4.35 \times 10^{-2}$ & $6.63 \times 10^{-2}$ & $6.62 \times 10^{-2}$ & $6.58 \times 10^{-2}$ & $2.46 \times 10^{-2}$ \\
${ }^{12} \mathrm{C}$ & $2.71 \times 10^{-2}$ & $2.25 \times 10^{-2}$ & $1.63 \times 10^{-2}$ & $2.07 \times 10^{-2}$ & $1.53 \times 10^{-2}$ \\
${ }^{16} \mathrm{O}$ & $8.74 \times 10^{-2}$ & $8.89 \times 10^{-2}$ & $7.85 \times 10^{-2}$ & $8.45 \times 10^{-2}$ & $4.72 \times 10^{-2}$ \\
${ }^{20} \mathrm{Ne}$ & $2.64 \times 10^{-3}$ & $2.74 \times 10^{-3}$ & $2.30 \times 10^{-3}$ & $2.38 \times 10^{-3}$ & $9.37 \times 10^{-2}$ \\
${ }^{24} \mathrm{Mg}$ & $2.58 \times 10^{-2}$ & $2.83 \times 10^{-2}$ & $2.66 \times 10^{-2}$ & $2.73 \times 10^{-2}$ & $1.05 \times 10^{-2}$ \\
${ }^{28} \mathrm{Si}$ & $1.56 \times 10^{-1}$ & $1.72 \times 10^{-1}$ & $1.69 \times 10^{-1}$ & $1.69 \times 10^{-1}$ & $9.89 \times 10^{-2}$ \\
${ }^{32} \mathrm{~S}$ & $9.55 \times 10^{-2}$ & $1.03 \times 10^{-1}$ & $1.03 \times 10^{-1}$ & $1.10 \times 10^{-1}$ & $6.35 \times 10^{-2}$ \\
${ }^{36} \mathrm{Ar}$ & $3.03 \times 10^{-2}$ & $3.23 \times 10^{-2}$ & $3.28 \times 10^{-2}$ & $3.19 \times 10^{-2}$ & $2.05 \times 10^{-2}$ \\
${ }^{40} \mathrm{Ca}$ & $4.39 \times 10^{-2}$ & $4.58 \times 10^{-2}$ & $4.62 \times 10^{-2}$ & $4.47 \times 10^{-2}$ & $3.03 \times 10^{-2}$ \\
${ }^{44} \mathrm{Ti}$ & $2.47 \times 10^{-2}$ & $3.46 \times 10^{-2}$ & $3.45 \times 10^{-2}$ & $3.42 \times 10^{-2}$ & $2.41 \times 10^{-2}$ \\
${ }^{48} \mathrm{Cr}$ & $3.38 \times 10^{-2}$ & $4.43 \times 10^{-2}$ & $4.37 \times 10^{-2}$ & $4.35 \times 10^{-2}$ & $3.58 \times 10^{-2}$ \\
${ }^{52} \mathrm{Fe}$ & $2.16 \times 10^{-2}$ & $2.46 \times 10^{-2}$ & $2.57 \times 10^{-2}$ & $2.56 \times 10^{-2}$ & $1.86 \times 10^{-2}$ \\
${ }^{56} \mathrm{Ni}$ & $3.66 \times 10^{-1}$ & $4.16 \times 10^{-1}$ & $4.36 \times 10^{-1}$ & $4.30 \times 10^{-1}$ & $2.02 \times 10^{-2}$ \\
${ }^{60} \mathrm{Zn}$ & $7.72 \times 10^{-4}$ & $1.12 \times 10^{-3}$ & $9.29 \times 10^{-4}$ & $9.08 \times 10^{-4}$ & $1.38 \times 10^{-1}$ \\
\end{tabular}

models $C_{1}$ and $D_{2}$ are of those having thinner helium envelopes. A practical reason lies behind the choice of models $B_{4,5,6}$ instead of models $\mathrm{B}_{1,2,3}$. The former ignite helium at larger densities amidst thicker envelopes, resulting in stronger helium detonations. The conditions after the collision at the antipodes are thus optimal to get the spontaneous detonation of the core, even when the simulations are carried out at a moderate resolution. We note, however, that the amount of ${ }^{56} \mathrm{Ni}$ synthesized during the $\mathrm{He}$ shell detonation in models $\mathrm{B}_{4,5,6}$ is an order of magnitude larger than in models $B_{1,2,3}$. This supports the current view that a necessary condition to reconcile SubCh-models with observations is to reduce the ignition density at the helium layer and/or the thickness of the accreted envelope. In this regard, models $\mathrm{C}$ and D are more suitable SN Ia progenitors. Even though these models have thinner envelopes than those of models B, they have more massive cores and larger central densities. This again facilitates the spontaneous detonation of carbon and its propagation through the core.

The complete explosion of the spherically symmetric model $A_{1}$ is in agreement with the evolution of similar models calculated by other groups. For example, the obtained Ni yield, $0.38 M_{\odot}$, is almost equal to that obtained by Moll \& Woosley (2013) for a similar model (their model A). The kinetic energy at $t \simeq 11.5 \mathrm{~s}$ is $\simeq 1.1 \times 10^{51} \mathrm{erg}$, completely compatible with a standard SN Ia explosion.

Several snapshots showing the detonation of the core of model $\mathrm{B}_{6}$ are depicted in the equatorial slice shown in Figures 10 and 11. A hot spot appears at the antipodes, when the He-shell ashes converge at $t \simeq 1.22 \mathrm{~s}$ (second snapshot). Nevertheless, the spontaneous detonation of the core still has to wait until $t \simeq 1.42 \mathrm{~s}$, the moment at which the compression waves arriving from the hot spot and from the center of the WD meet (see the third snapshot in Figure 11). After this moment, a steady detonation forms and propagates inwards through the core (first and second snapshots in the second row). Meanwhile, the rotation of the core between the first and fifth snapshots is clearly visible. Finally, the whole core has been burned at the last snapshot at $t=2.03 \mathrm{~s}$. The detonation of the core in models $\mathrm{B}_{4}$ and $\mathrm{B}_{5}$ follow a qualitatively similar path. The complete detonation of the more massive WDs, models $\mathrm{C}_{1}$ and $\mathrm{D}_{2}$, proceed in a similar way, 


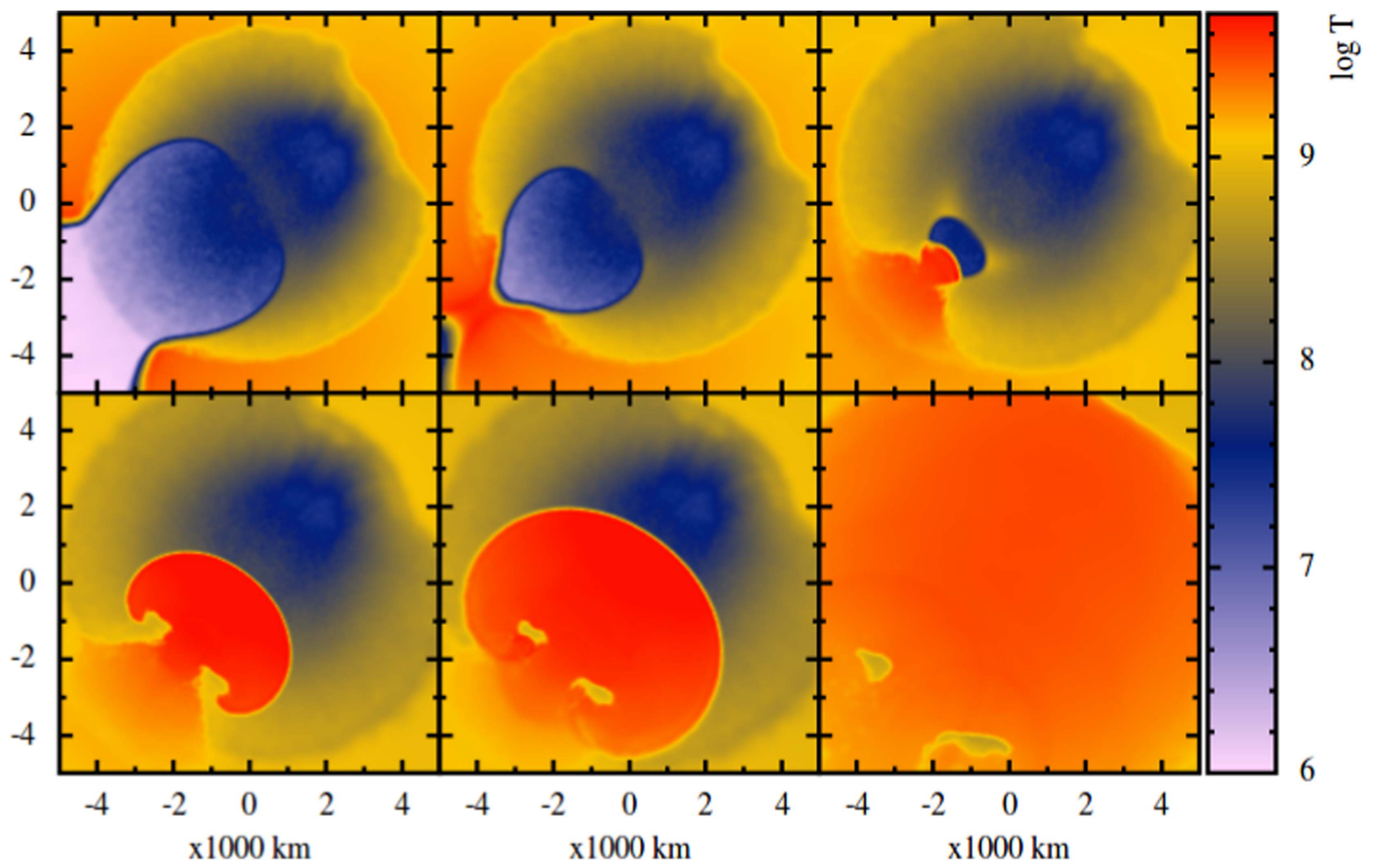

Figure 10. Colormap of temperature in a $Y Z$ (equatorial) slice, showing the core detonation of model $\mathrm{B}_{6}$ in Table 1 at times $t=1.10,1.22,1.42,1.62,1.72$, and $2.03 \mathrm{~s}$, respectively. The box size is $[-5: 5] \times 10^{3} \mathrm{~km}$ in all directions.

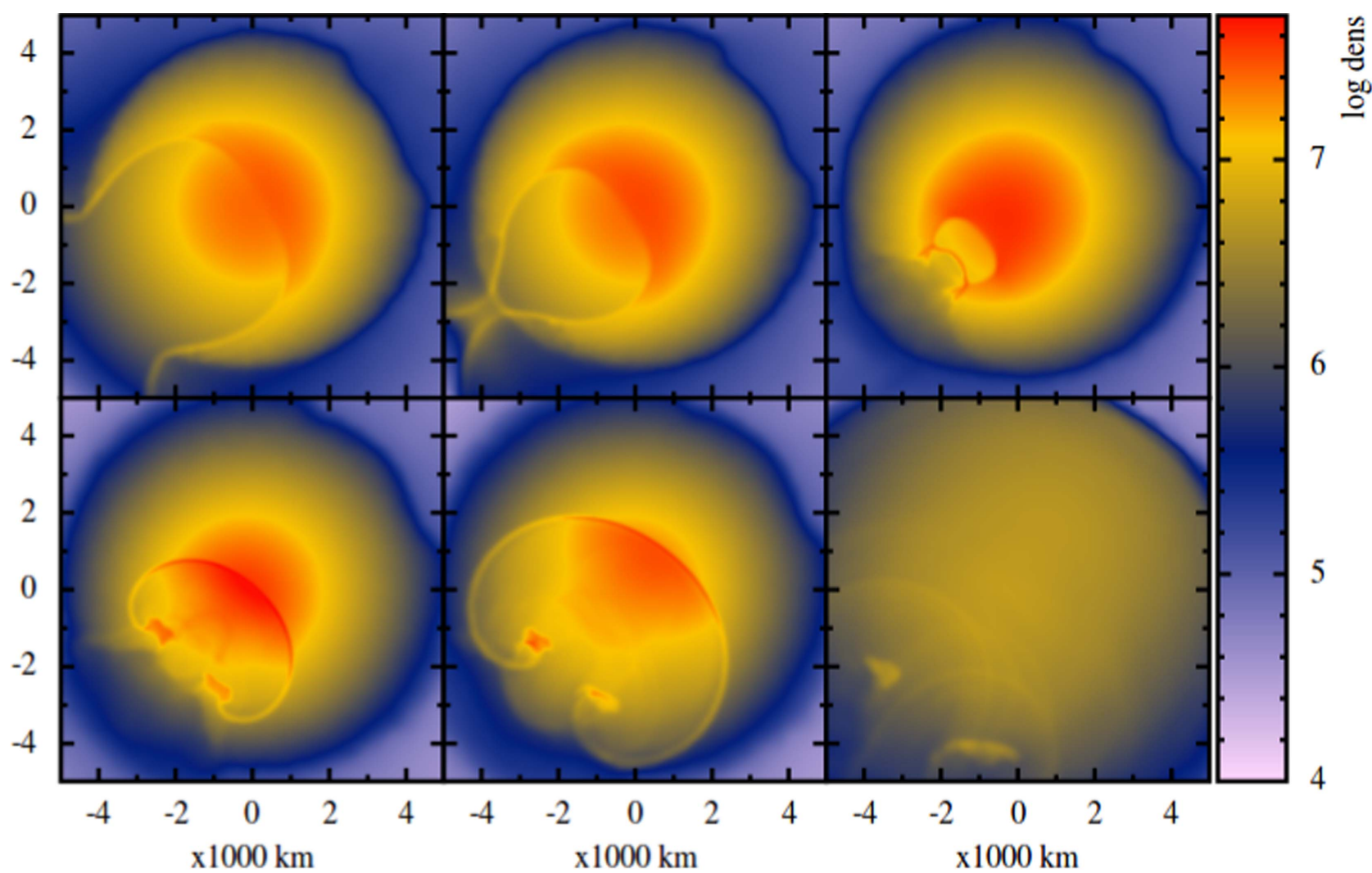

Figure 11. Same as Figure 10, but for density. 

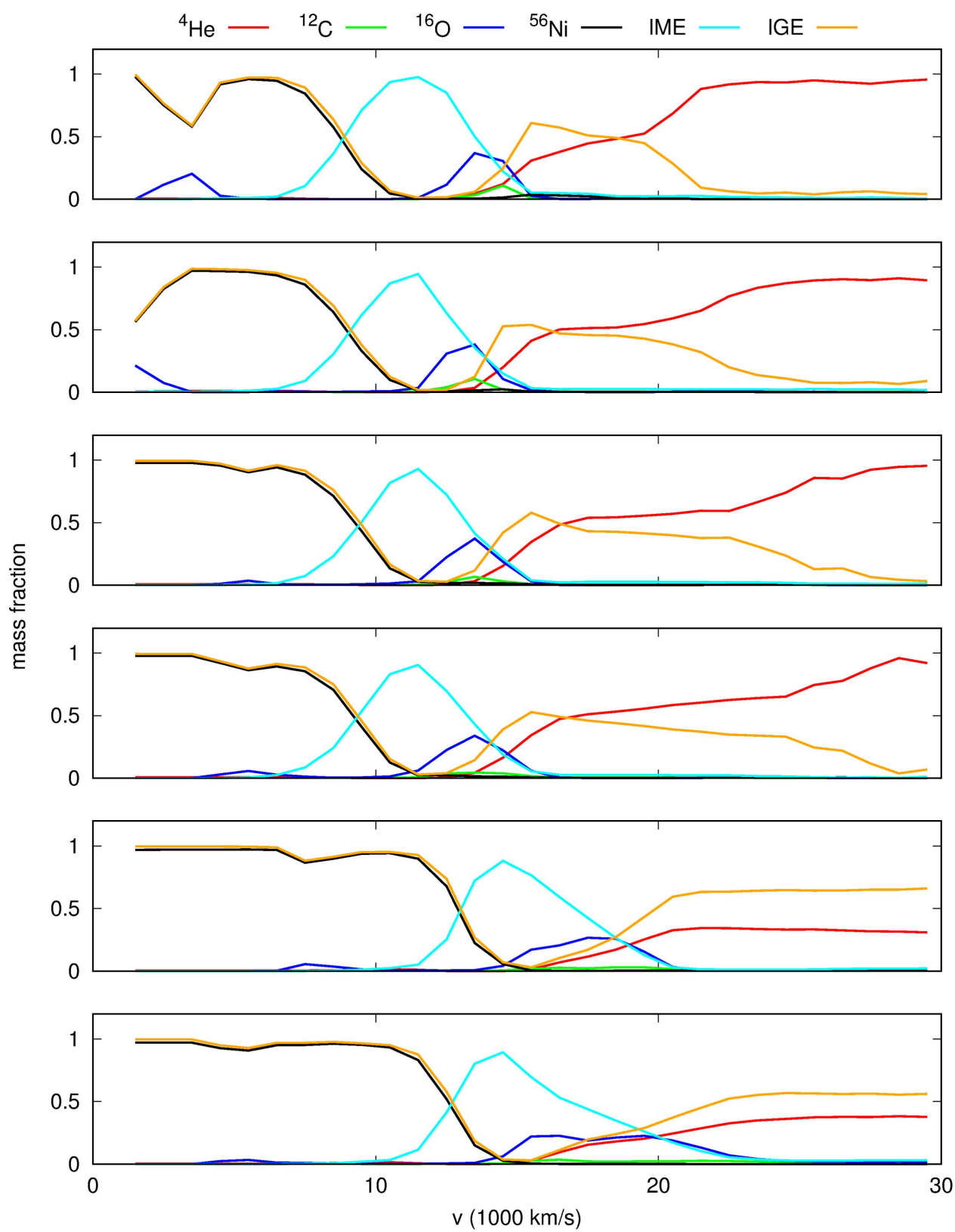

Figure 12. Complete detonation of the WD: mass fractions of the main groups of nuclei in velocity space at $t=11.5 \mathrm{~s}$. From top to bottom: models $\mathrm{A}_{1}, \mathrm{~B}_{4}, \mathrm{~B}_{5}, \mathrm{~B}_{6}$, $\mathrm{C}_{1}$, and $\mathrm{D}_{2}$ (see Tables 1 and 5).

although they produce a larger amount of ${ }^{56} \mathrm{Ni}$ and the explosion is, consequently, stronger (Table 4).

Because the CO core is incinerated supersonically, we do not expect large differences in the energetics or in the ejected nuclear yields among the rotating models and, in fact, this is what our simulations show (Tables 4 and 5). There are, however, several differences in the product yields of the explosion with respect those of the spherically symmetric models $A_{1}$ and $C_{1}$. While the amount of IMEs is only slightly larger in the less massive rotating models $\mathrm{B}_{4,5,6}$, which can be explained by the larger mass of the WD, the ${ }^{8}$ IGEs are comparatively more copiously produced. This is a different trend from that found in rotating Chandrasekhar- and super-Chandrasekhar-mass models igniting at much higher densities. In those models, a fast rotation favors

\footnotetext{
8 We have grouped all nuclei between ${ }^{20} \mathrm{Ne}$ and ${ }^{40} \mathrm{Ca}$ as IMEs and those from ${ }^{44} \mathrm{Ti}$ up to ${ }^{60} \mathrm{Zn}$ as IGEs.
}

the production of IMEs (Pfannes et al. 2010a, 2010b). On the other hand, the more massive rotating model $\mathrm{D}_{2}$ gives a more balanced increase in the IME and IGE production compared to its non-rotating counterpart, model $\mathrm{C}_{1}$. The enhanced production of IGEs in models $\mathrm{B}_{4}, \mathrm{~B}_{5}$, and $\mathrm{B}_{6}$ with respect to the nonrotating model $A_{1}$ is due to their larger core and He-shell masses. Having a thicker He-envelope tamper increases the average density of the core at the moment of carbon detonation, which ultimately favors the production of heavy nuclei. Because of the higher production of IGE, the kinetic energy of the explosion is consequently larger in the rotating models, $\simeq 1.25 \times 10^{51}$ erg $($ models $\mathrm{B})$ and $\simeq 1.5 \times 10^{51} \mathrm{erg}$ (model $\mathrm{D}_{2}$ ). The distribution of the abundances in velocity space around $t=11.5 \mathrm{~s}$ is depicted in Figure 12. Among the models with a thicker He envelope, the most relevant feature is that the IGE profiles (orange lines) spread to larger velocities in the rotating models, especially in the oblique igniters $\mathrm{B}_{5}$ and $\mathrm{B}_{6}$. The 
abundance distribution in models $\mathrm{C}_{1}$ and $\mathrm{D}_{2}$, with more massive cores and thinner envelopes, is shifted to higher velocities. For example, the well-defined peak in the mass fraction of IMEs is shifted $\simeq 2500 \mathrm{~km} . \mathrm{s}^{-1}$ with respect models B. Also note that the high-velocity tail of the ejecta is dominated by ${ }^{44} \mathrm{Ti},{ }^{48} \mathrm{Cr}$, and ${ }^{52} \mathrm{Fe}$, while the ${ }^{56} \mathrm{Ni}$ is actually moving with velocities lower than $15,000 \mathrm{~km} \mathrm{~s}^{-1}$.

\section{Conclusions}

In this work, we addressed the question of the fate of rotating WDs that detonate helium at the base of an accreted shell, when their masses are well below the Chandrasekhar-mass limit. A study of this kind, which has never been attempted before, is pertinent for several reasons. The most compelling of these being that in a spinning WD, the location of the initial kernel/s leading to helium-shell detonation are not necessarily located on the rotation axis. Thus, the strong (almost point-like) convergence of the ashes of the He detonation on the antipodes of the ignition region, typical of spherically symmetric models, is lost. Such loss of focusing in the convergence of the ashes changes the physical conditions at the underlying carbon core, which may be less prone to detonate. A second goal was to make a comparison among the main observables coming from both the rotating and non-rotating models. To do that, we have considered two potential explosion scenarios. In the first case, the secondary carbon detonation was artificially suppressed, and the main observables of the subluminous event, produced by the He-shell detonation, were determined. In the second case, carbon was allowed to detonate, which, according to our own results is the most plausible outcome. Again, the main observables were obtained and compared with a non-rotating spherically symmetric model.

The rotational velocity of an accreting WD is set by the total amount of accreted material, by the efficiency of angular momentum transport from the surface to the core, and by the angular momentum losses. In the case of the DDet scenario, the mass of the accreted layer is not as large as in the Chandrasekhar-mass models of SNe Ia, and the ensuing angular velocity is expected to be lower. The precise profile of the angular velocity in the progenitor of subCh-mass explosion models is not well known (see Section 2). On a practical basis, we have adopted rigid rotation, which facilitates building rotating equilibrium models with the SPH technique, and is a realistic hypothesis in the case of efficient angular momentum transport. In any case, our simulations aim to study how the propagation of helium detonation is affected by a change in the geometry of the He shell and the CO-core interface. Assuming rigid rotation is enough to conduct such exploratory study.

As a principal result, we confirm the robustness of the DDet mechanism as a viable scenario to give rise to SN Ia explosion. According to our results, igniting helium far from the rotational axis blurs the convergence of the detonation to the antipodes, as expected. But, rather than hindering it, the slight asynchronicity in the arrival of the detonation waves seems to enhance the chances of inducing carbon detonation below the $\mathrm{CO}$ core (see Figure 4). When the helium initially detonates close to the rotational axis, the geometrical focusing at the antipodes is preserved, and the results are similar to those of the spherically symmetric model. These results also hold for smaller helium shells, $\simeq 0.05 M_{\odot}(\mathrm{D}$ models in Table 1$)$.

We have carried out a separate study of both the detonation of the He shell alone and the combined He-shell and CO-core detonations. The former case would give rise to a peculiar subluminous SN Ia event, in which the light curve is powered by radioactive ${ }^{48} \mathrm{Cr}$ and ${ }^{52} \mathrm{Fe}$, with a minor contribution of ${ }^{56} \mathrm{Ni}$. Nevertheless, we found that the precise yield of ${ }^{56} \mathrm{Ni}$ is very dependent on the density at the base of the He shell at the moment of explosion. The radioactive ${ }^{44} \mathrm{Ti}$ seems to be more copiously produced in rotating WDs. The column density map of the radioactive elements produced in the explosion of the spinning models shows a larger loss of spherical symmetry than in the non-rotating case (see Figure 8). Such asymmetry might increase the polarization signatures of the spectra, which is low in standard non-rotating subCh-mass models (Bulla et al. 2016). Nonetheless, this qualitative result has to be confirmed with more detailed calculations of the polarization spectra.

When the ${ }^{12} \mathrm{C}+{ }^{12} \mathrm{C}$ reaction is allowed to proceed, the detonation of the $\mathrm{He}$ shell is always followed by the spontaneous detonation of the core. A robust explosion, energetically compatible with a standard SN Ia event, is obtained in all the cases studied. However, the rotating models with thicker He envelopes do show enhanced production of IGEs, some of them moving at a high velocity during the homologous expansion. The large amount of IGEs moving at $v \geqslant 2 \times 10^{4} \mathrm{~km} \mathrm{~s}^{-1}$, besides the expected increase in the polarization signatures of the explosion, conspire against fast spinning WDs with thick helium layers as viable progenitors of $\mathrm{SNe}$ Ia. A reduction in the mass of the accreted He shell would help with this problem. We have shown that halving the mass of the helium envelope still leads to the detonation of the core in spinning WDs ( $\mathrm{C}$ and $\mathrm{D}$ models in Table 1). Nevertheless, reducing the mass of the accreted envelope also lowers the amount of angular momentum gained by the WD. For He-shell masses $\leqslant 0.01 M_{\odot}$, the geometry of the WD would remain almost spherical.

The combination of a low-mass He shell on top of an oblate substrate made of carbon and oxygen may, however, be realized in the DD scenario (Guillochon et al. 2010; Dan et al. 2015). It has been suggested that the DDet mechanism, postulated to explain the subCh-mass route to $\mathrm{SNe}$ Ia, could also be at work in the DD scenario (Pakmor et al. 2013). In this regard, the results presented in this paper can also be useful to better understand the DD scenario of SNe Ia.

We acknowledge useful comments by Stan Woosley and by the anonymous referee. This work has been supported by the MINECO Spanish project AYA2017-86274-P and The Generalitat of Catalonia SGR-661/2017 (D.G.), by the Swiss Platform for Advanced Scientific Computing (PASC) project SPH-EXA (R.C. and D.G.) and by the Spanish MINECO-FEDER project AYA2015-63588-P (I.D.). The authors acknowledge the support of the sciCORE (http:// scicore.unibas.ch/) scientific computing core facility at the University of Basel, where some of the calculations were performed. D.G. acknowledges the support and hospitality provided by the Plasma Physics Group at the Imperial College in London, during the revision process of this manuscript.

\section{Appendix Implementation of Rotation}

An accurate method to build rotating WDs in hydrostatic equilibrium within the SPH framework does not exist. We have 
Table 6

Rotation Features of the White Dwarfs After Relaxation

\begin{tabular}{|c|c|c|c|c|c|c|c|c|c|}
\hline Model & $\begin{array}{c}\text { Mass } \\
M_{\odot}\end{array}$ & $\begin{array}{c}J \\
10^{50} \mathrm{erg} \mathrm{s}\end{array}$ & $\mathrm{s}^{-1}$ & $\begin{array}{c}W \\
10^{50} \mathrm{erg}\end{array}$ & $\begin{array}{c}T \\
10^{50} \mathrm{erg}\end{array}$ & $\begin{array}{c}U \\
10^{50} \mathrm{erg}\end{array}$ & $T /|W|$ & $\begin{array}{l}R_{e} \\
\mathrm{~km}\end{array}$ & $R_{p} / R_{e}$ \\
\hline B & 1.081 & 0.798 & 0.500 & -4.805 & 0.200 & 2.710 & 0.042 & 8000 & 0.650 \\
\hline
\end{tabular}

Note. Main features of the rigid rotators B and D of Table 1 at equilibrium. Symbols $J$ and $\omega$ are the total angular momentum and the angular velocity, whereas $W$, $T$, and $U$ are the total gravitational, kinetic, and internal energies. The last two columns show the equatorial radius $R_{e}$ and the polar to equatorial radii ratio, respectively.

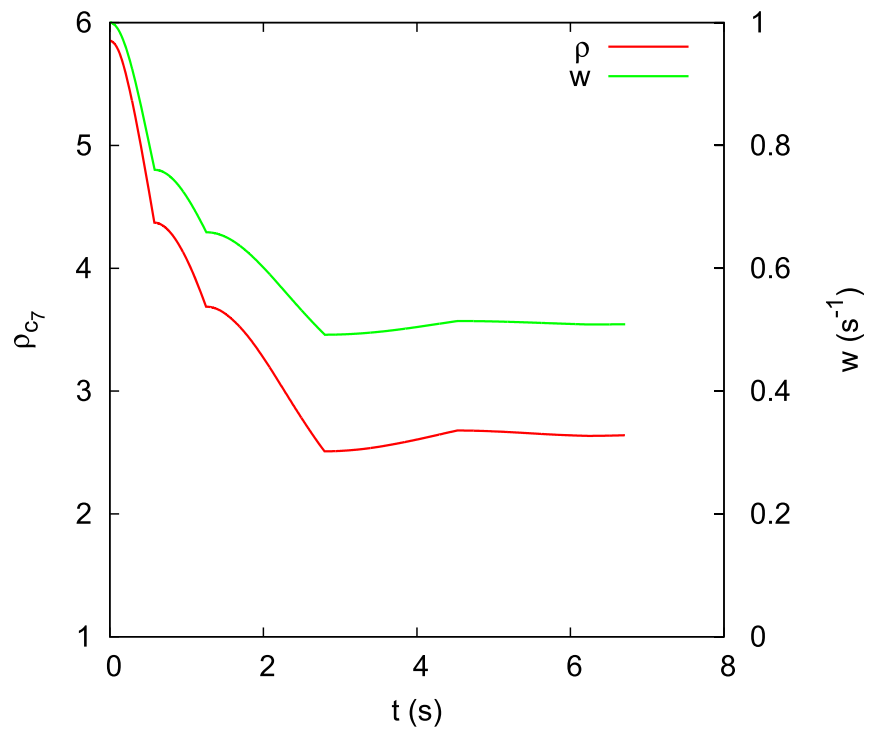

Figure 13. Example of a relaxation sequence toward equilibrium. The total mass $M=1.081 M_{\odot}$ and total angular momentum $J=0.798 \times 10^{50} \mathrm{erg} \mathrm{s}$ were kept constant, while the angular velocity and central density evolve to achieve stable values. The locations where the slope of the curves change indicate the times at which the particle velocities are set to zero to remove numerical noise.

developed and checked a relaxation procedure that is able to produce self-gravitating rotating structures in equilibrium. We assume that rotation is axisymmetric and that any physical and geometrical features of the oblate structure in equilibrium is basically determined by the total mass $M_{\mathrm{WD}}$ and total angular momentum $J_{\mathrm{WD}}$. Both magnitudes, $M_{\mathrm{WD}}$ and $J_{\mathrm{WD}}$, are specified at $t=0$ and kept constant during the relaxation process, during which we let the sample of SPH mass points evolve under the self-gravity and the centripetal force in a corotating frame. After several sound-crossing times, the rotating structures come to an equilibrium.

Starting from a spherically symmetric model of a WD with central density $\rho_{0}$, a sample of $N$ mass particles is spread according to the density profile $\rho(r)$. The distribution in the spherical angles $\phi$ and $\theta$ is chosen at random. We introduce the rigid rotation as a fictitious centripetal force, which is added to the gravity $\boldsymbol{f}_{\boldsymbol{c}}=-\omega(t) \times(\omega(t) \times \boldsymbol{r}(t))$, where $\boldsymbol{\omega}(t)=\omega_{x}(t) \hat{\boldsymbol{i}}$ is the angular velocity at the elapsed time $t$ (the $X$-axis has been assumed as the rotation axis in this work) and $\boldsymbol{r}(t)$ is the position vector of the particle. The angular momentum of this configuration, as viewed from an inertial reference frame, is $J_{x}=I_{x x} \omega_{x}$, where $I_{x x}$ is the moment of inertia around the rotation axis. We let this configuration free to evolve and compute the time-dependent angular velocity of the WD at each integration step, so that the total angular momentum is preserved $\omega_{x}(t)=\frac{I_{x x}(t=0)}{I_{x x}(t)} \omega_{x}(t=0)$. The velocity of the particles is regularly set to zero to remove spurious numerical noise. A typical relaxation sequence is shown in Figure 13, where we see how the $\omega(t)$ and the central density $\rho(t)$ approach stable values after several seconds of evolution. A summary of the equilibrium rotation features of the WDs used in this work is provided in Table 6.

\section{ORCID iDs}

D. García-Senz (i) https://orcid.org/0000-0001-5197-7100

R. M. Cabezón (1i) https://orcid.org/0000-0003-3546-3964

I. Domínguez (i) https://orcid.org/0000-0002-3827-4731

\section{References}

Badenes, C., \& Maoz, D. 2012, ApJL, 749, L11

Bildsten, L., Shen, K. J., Weinberg, N. N., \& Nelemans, G. 2007, ApJL, 662, L95

Blinnikov, S. I., Dunina-Barkovskaya, N. V., \& Nadyozhin, D. K. 1996, ApJS, 106, 171

Blondin, S., Dessart, L., Hillier, D. J., \& Khokhlov, A. M. 2017, MNRAS, 470,157

Bulla, M. 2017, PhD thesis, Queen's Univ.

Bulla, M., Sim, S. A., Kromer, M., et al. 2016, MNRAS, 462, 1039

Cabezón, R. M., García-Senz, D., \& Bravo, E. 2004, ApJS, 151, 345

Cabezón, R. M., García-Senz, D., \& Figueira, J. 2017, A\&A, 606, A78

Cabezón, R. M., García-Senz, D., \& Relaño, A. 2008, JCoPh, 227, 8523

Dan, M., Guillochon, J., Brüggen, M., Ramirez-Ruiz, E., \& Rosswog, S. 2015, MNRAS, 454, 4411

Fink, M., Hillebrandt, W., \& Röpke, F. K. 2007, A\&A, 476, 1133

Fink, M., Röpke, F. K., Hillebrandt, W., et al. 2010, A\&A, 514, A53

García-Senz, D., \& Bravo, E. 2005, A\&A, 430, 585

García-Senz, D., Bravo, E., \& Woosley, S. E. 1999, A\&A, 349, 177

García-Senz, D., Cabezón, R. M., Domínguez, I., \& Thielemann, F. K. 2016, ApJ, 819, 132

García-Senz, D., Cabezón, R. M., \& Escartín, J. A. 2012, A\&A, 538, A9

Ghosh, P., \& Wheeler, J. C. 2017, ApJ, 834, 93

Goldstein, D. A., \& Kasen, D. 2018, arXiv:1801.00789

Guillochon, J., Dan, M., Ramirez-Ruiz, E., \& Rosswog, S. 2010, ApJL, 709, L64

Hillebrandt, W., Kromer, M., Röpke, F. K., \& Ruiter, A. J. 2013, FrPhy, 8, 116

Hillebrandt, W., \& Niemeyer, J. C. 2000, ARA\&A, 38, 191

Hoeflich, P., Hsiao, E. Y., Ashall, C., et al. 2017, ApJ, 846, 58

Hoeflich, P., Khokhlov, A., Wheeler, J. C., et al. 1996, ApJL, 472, L81

Holcomb, C., Guillochon, J., De Colle, F., \& Ramirez-Ruiz, E. 2013, ApJ, 771,14

Iben, I., Jr., \& Tutukov, A. V. 1984, ApJS, 54, 335

Jiang, J.-A., Doi, M., Maeda, K., et al. 2017, Natur, 550, 80

Khokhlov, A. M. 1991, A\&A, 245, 114

Langer, N., Deutschmann, A., Wellstein, S., \& Höflich, P. 2000, A\&A 362,1046

Livne, E., \& Arnett, D. 1995, ApJ, 452, 62

Livne, E., \& Glasner, A. S. 1991, ApJ, 370, 272

Lorén-Aguilar, P., Isern, J., \& García-Berro, E. 2009, A\&A, 500, 1193

Maeder, A., \& Meynet, G. 2000, ARA\&A, 38, 143

Maoz, D., Mannucci, F., \& Nelemans, G. 2014, ARA\&A, 52, 107 
Mereghetti, S. 2015, in Thirteenth Marcel Grossmann Meeting: On Recent Developments in Theoretical and Experimental General Relativity, Astrophysics and Relativistic Field Theories, ed. K. Rosquist (Singapore: World Scientific), 2459

Moll, R., \& Woosley, S. E. 2013, ApJ, 774, 137

Moore, K., Townsley, D. M., \& Bildsten, L. 2013, ApJ, 776, 97

Neunteufel, P., Yoon, S.-C., \& Langer, N. 2017, A\&A, 602, A55

Niemeyer, J. C., \& Woosley, S. E. 1997, ApJ, 475, 740

Nomoto, K., Thielemann, F.-K., \& Yokoi, K. 1984, ApJ, 286, 644

Nugent, P., Baron, E., Branch, D., Fisher, A., \& Hauschildt, P. H. 1997, ApJ, 485,812

Pakmor, R., Kromer, M., Taubenberger, S., \& Springel, V. 2013, ApJL, 770, L8 Patterson, J. 1980, ApJ, 241, 235

Pfannes, J. M. M., Niemeyer, J. C., \& Schmidt, W. 2010a, A\&A, 509, A75

Pfannes, J. M. M., Niemeyer, J. C., Schmidt, W., \& Klingenberg, C. 2010b, A\&A, 509, A74

Phillips, M. M. 1993, ApJL, 413, L105

Phillips, M. M., Lira, P., Suntzeff, N. B., et al. 1999, AJ, 118, 1766

Piro, A. L. 2008, ApJ, 679, 616

Plewa, T., Calder, A. C., \& Lamb, D. Q. 2004, ApJL, 612, L37
Popov, S. B., Mereghetti, S., Blinnikov, S. I., Kuranov, A. G., \& Yungelson, L. R. 2018, MNRAS, 474, 2750

Price, D. J. 2007, PASA, 24, 159

Ritter, H. 1985, A\&A, 148, 207

Ruiter, A. J., Belczynski, K., Sim, S. A., et al. 2011, MNRAS, 417, 408

Saio, H., \& Nomoto, K. 2004, ApJ, 615, 444

Seitenzahl, I. R., Meakin, C. A., Townsley, D. M., Lamb, D. Q., \& Truran, J. W. 2009, ApJ, 696, 515

Shen, K. J., \& Moore, K. 2014, ApJ, 797, 46

Sim, S. A., Fink, M., Kromer, M., et al. 2012, MNRAS, 420, 3003

Sim, S. A., Röpke, F. K., Hillebrandt, W., et al. 2010, ApJL, 714, L52

Sim, S. A., Sauer, D. N., Röpke, F. K., \& Hillebrandt, W. 2007, MNRAS, 378,2

Wang, L., \& Wheeler, J. C. 2008, ARA\&A, 46, 433

Whelan, J., \& Iben, I., Jr. 1973, ApJ, 186, 1007

Woosley, S. E., \& Kasen, D. 2011, ApJ, 734, 38

Woosley, S. E., Taam, R. E., \& Weaver, T. A. 1986, ApJ, 301, 601

Woosley, S. E., \& Weaver, T. A. 1994, ApJ, 423, 371

Yoon, S.-C., \& Langer, N. 2004a, A\&A, 419, 645

Yoon, S.-C., \& Langer, N. 2004b, A\&A, 419, 623 